\title{
Stochastic computing in convolutional neural network implementation: A review
}

\author{
Yang Yang Lee ${ }^{1}$, Zaini Abdul Halim ${ }^{\text {Corresp. } 1}$ \\ ${ }^{1}$ School of Electrical and Electronic Engineering, Universiti Sains Malaysia, Nibong Tebal, Penang, Malaysia \\ Corresponding Author: Zaini Abdul Halim \\ Email address: zaini@usm.my
}

Stochastic computing (SC) is an alternative computing domain for ubiquitous deterministic computing whereby a single logic gate can perform the arithmetic operation by exploiting the nature of probability math. SC was proposed in the 1960s when binary computing was expensive. However, presently, SC started to regain interest after the widespread of deep learning application, specifically the convolutional neural network (CNN) algorithm due to its practicality in hardware implementation. Although not all computing functions can translate to the SC domain, several useful function blocks related to the CNN algorithm had been proposed and tested by researchers. An evolution of CNN, namely, binarised neural network, had also gained attention in the edge computing due to its compactness and computing efficiency. This study reviews various SC CNN hardware implementation methodologies. Firstly, we review the fundamental concepts of SC and the circuit structure and then compare the advantages and disadvantages amongst different SC methods. Finally, we conclude the overview of SC in CNN and make suggestions for widespread implementation. 


\section{Stochastic Computing in Convolutional Neural}

2 Network Implementation: A Review

3

4

5

6

\section{7}

8

9

10

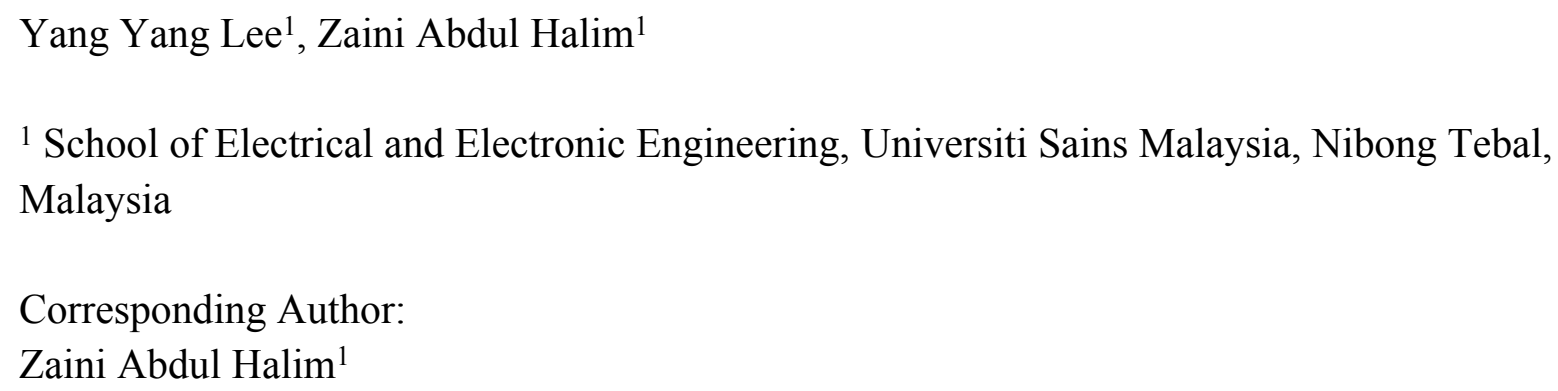

\section{Abstract}

Stochastic computing (SC) is an alternative computing domain for ubiquitous deterministic computing whereby a single logic gate can perform the arithmetic operation by exploiting the nature of probability math. SC was proposed in the 1960 s when binary computing was expensive. However, presently, SC started to regain interest after the widespread of deep learning application, specifically the convolutional neural network (CNN) algorithm due to its practicality in hardware implementation. Although not all computing functions can translate to the SC domain, several useful function blocks related to the $\mathrm{CNN}$ algorithm had been proposed and tested by researchers. An evolution of CNN, namely, binarised neural network, had also gained attention in the edge computing due to its compactness and computing efficiency. This study reviews various SC CNN hardware implementation methodologies. Firstly, we review the fundamental concepts of $\mathrm{SC}$ and the circuit structure and then compare the advantages and disadvantages amongst different SC methods. Finally, we conclude the overview of SC in CNN and make suggestions for widespread implementation.

\section{Nomenclature}

$\begin{array}{llll}\text { ADC } & \text { analogue-to-digital converter } & \text { FPU } & \text { floating-point unit } \\ \text { ALU } & \text { arithmetic logic unit } & \text { FSM } & \text { finite state machine } \\ \text { APC } & \text { approximate parallel counter } & \text { GPU } & \text { graphic processing unit } \\ \text { ASC } & \text { analogue-to-stochastic converter } & \text { IoT } & \text { internet of things } \\ \text { ASIC } & \text { application-specific integrated circuit } & \text { LFSR } & \text { linear feedback shift register } \\ \text { BNN } & \text { binarised neural network } & \text { MAC } & \text { multiplier-accumulator } \\ \text { Btanh } & \text { binary input Stanh } & \text { ReLU } & \text { rectified linear unit } \\ \text { CNN } & \text { convolutional neural network } & \text { RNG } & \text { random number generator } \\ \text { CPU } & \text { central processing unit } & \text { SC } & \text { stochastic computing } \\ \text { DNN } & \text { deep neural network } & \text { SNG } & \text { stochastic number generator } \\ \text { DWM } & \text { domain-wall memory } & \text { SUC } & \text { shifted unary code }\end{array}$



EDT early decision termination
Stanh stochastic TanH
FA full adder
TanH hyperbolic tangent
FPGA field-programmable gate array
WBG weighted binary generator

\section{Introduction}

Deep learning algorithms had been widely and silently integrated into our daily life, for example, image enhancer, voice search and linguistic translation. Meanwhile, the Internet of things (IoT) had gained industrial recognition, and many applications rely on edge computing whereby data are processed on the fly rather than relayed to cloud computing for reliability and security reasons(Naveen \& Kounte, 2019). People had been heavily dependent on a widely accessible central processing unit (CPU) and general-purpose graphics processing unit (GPU) for deep learning research and application deployment. Although users strive to achieve great real-time response by offloading many computationally intensive tasks, such as object recognition to edge devices, those computing devices become extremely inefficient despite the utmost priority of power efficiency in IoT. Although the field-programmable gate array (FPGA) and applicationspecific integrated circuit (ASIC) could overcome the power efficiency issue, economically implementing deep learning hardware logic is not ideal. Thus, researchers are trying to explore alternatives to conventional binary in this specific use case, driving the rise of stochastic computing (SC).

SC was proposed in the 1960s when the cost of implementing binary computing was prohibitive but soon ran out of favour in the semiconductor industry. Unlike binary computing, $\mathrm{SC}$ can perform the arithmetic operation with a single logic gate. The most evident advantage of $\mathrm{SC}$ is its ability to reduce the area and power draw by reducing the number of active transistors (De Aguiar \& Khatri, 2015). SC is also an inherently progressive precision where the output converges from the most significant figure; thus, $\mathrm{SC}$ is capable of making early decision termination (EDT). Power efficiency and EDT capability make deep learning application favourable (Kim et al., 2016), particularly in convolutional neural network (CNN) application.

CNN received extensive development since its introduction in 2012 due to its unprecedented performance in object recognition. CNN model development was trending from being deep and massive (highly accurate) to responsive (fast inference). In response to the IoT requirements in edge computing, researchers had attempted to reduce the math precision to save computing resources. With a reasonable trade-off for accuracy, an extreme simplification version of CNN, that is, binarised neural network (BNN), emerged with a promising hardware implementation capability and computing efficiency, rivalling SC methodology.

$\mathrm{SC}$ in CNN lacks widespread attention due to its cross-disciplinary nature in the computer science study. CNN is impactful in the field of machine learning, but the rising of IoT edge computing which pursues efficient computing pushed back CNN implementation hard. While many researchers focus on innovating CNN algorithms for different use cases such as medicine and agriculture, only a few of them consider how to implement CNN realistically since CNN execution is computationally intensive by itself. Given that no comprehensive and updated 
68

69

70

71

72

73

74

75

76

77

78

79

80

81

82

83

84

85

86

87

88

89

90

91

92

93

94

95

96

97

98

99

100

101

102

103

104

105

106

107

review exists on this specific area, thus, in this review paper, we attempt to investigate and survey the $\mathrm{SC}$ implementation in the $\mathrm{CNN}$ application.

\section{Review Methodology}

This review intends to answer the following research questions:

1) What are the major developments of SC elements and $\mathrm{SC} C N N$ in recent years? Due to the narrow field of study in SC, the related studies are scattered, let alone the SC in $\mathrm{CNN}$ implementation; thus, impede the development of SC CNN without a more centralised reference, increasing difficulty in identifying the research trends.

2) How exactly is the CNN being computed/executed in the stochastic domain? $\mathrm{SC}$ is a unique computing methodology which is not often being mentioned in the academic study, despite its unique advantage in the surge of CNN application. Thus, there is a need to have a big picture on the SC CNN mechanism.

3) What are the open problems and/or opportunities to implement SC CNN? SC CNN does have its implementation hurdles. Thus, it is necessary to summarise them before moving forward in this field of research.

With the research questions in mind, we first reviewed the basic concepts of SC and CNN in modern perspectives. It is necessary to understand the background of SC and CNN due to the vastly different field of studies between them. Moreover, there is a need to aggregate the knowledge of SC elements in the face of rising trends in SC developments. Then we examined the recent developments and contributions of SC in CNN computation and compared the implementation methodologies across various recent studies. Finally, we made a conclusion and some suggestions for the future of deep learning research in the SC domain.

\section{Search criteria}

An initial search was carried out to identify an initial set of papers which have the prior works on $\mathrm{SC}$ and $\mathrm{CNN}$ in hardware implementation. The search strings were then inferred and developed as follows:

('Stochastic computing') OR ('Stochastic computing deep learning') OR ('Stochastic computing convolutional neural network') OR ('Stochastic computing neural network') OR ('Stochastic computing image processing')

'Stochastic' alone has a lot of meaning in a wide area of study. Thus, the keyword of 'Stochastic computing' is a necessity to narrow down the search scope. The search strings were applied to the indexed scientific database Scopus and Web of Science (peer-reviewed). Domainoriented databases (ACM Digital Library, IEEE Xplore Digital Library) were also referred extensively. Finally, Google Scholar (limited to peer-reviewed papers) were used to find any omitted academic literature, especially in this multi-disciplinary search scope. Peer-reviewed 
108

109

110

111

112

113

114

115

116

117

118

119

120

121

122

123

124

125

126

127

128

129

130

131

132

133

134

135

136

137

138

139

140

141

142

143

144

145 paper.

\section{Scope of review}

\section{Basic Concepts}

\section{SC} frequency of 1's bit, as shown in Fig. 1.

\section{Figure 1 here} operation, the output can be defined as:

$$
\mathrm{S}_{3}=\mathrm{P}\left(\mathrm{S}_{3}\right)=\mathrm{P}\left(\mathrm{S}_{1}\right) \mathrm{P}\left(\mathrm{S}_{2}\right)=\mathrm{S}_{1} \times \mathrm{S}_{2} \text {. }
$$

articles were preferred to ensure only confirmed works were to be summarised in this review

Notably, SC is not the only methods existed for efficient CNN computing. We only cover the topics of SC and SC related to CNN computing in this review study. Many articles may not directly involve $\mathrm{CNNs}$, but their novel SC elements are worthy as part of the significant SC developments and potentially useful for the future SC CNN function blocks, thus, will be mentioned in this review. Some elemental studies on CNNs were referred to understand the nature of $\mathrm{CNN}$ algorithms better. Some surveys on CNN implementation in FPGA merely or never discuss the SC, but they shared a similar concern on efficient $\mathrm{CNN}$ computation. Thus, their surveys were also considered and referred to in this review study if any.

$\mathrm{SC}$ and $\mathrm{CNN}$ are different fields of studies and worth a separate explanation. Thus, SC will be described first, then secondly $\mathrm{CNN}$ and $\mathrm{BNN}$ will be explained. Lastly, the competitive relationship between SC and BNN implementations will be discussed. SC is a unique concept of computing relative to traditional binary computing and has to be understood before an in-depth discussion on $\mathrm{SC}$ implementation in $\mathrm{CNN}$ at the next section.

$\mathrm{SC}$ is favourable in IoT application due to its extreme simplicity of computing elements, where power efficiency is of utmost priority. Unlike deterministic computing that tolerates absolutely no error, SC allows errors to a certain degree, thus the name approximate computing. SC initially decodes a binary number into a bitstream in such a way that the frequency of 1's bit represents the magnitude of value. For example, $[0,0,0,0,0,1,1,1]$ stochastic stream is equal to $3 / 8$ or 0.375 because it has three 1 's bits. Then, the number can be computed in the stochastic domain with a simple logic gate instead of gate combinations in the binary domain. Finally, the stochastic stream will be converted back to binary numbers with a simple counter by counting the

SC took advantage of probability math to reduce the logic components required to perform an arithmetic operation. Taking Fig. 2(A) and (B) as examples, in the AND gate multiplication

In the case of addition operation, the output will be scaled by half with MUX select input with a bitstream value of 0.5 . The MUX scaled adder can be defined as: 
$\mathrm{S}_{4}=\mathrm{P}\left(\mathrm{S}_{3}\right) \mathrm{P}\left(\mathrm{S}_{1}\right)+\left(1-\mathrm{P}\left(\mathrm{S}_{3}\right)\right) \mathrm{P}\left(\mathrm{S}_{2}\right)=\frac{\mathrm{P}\left(\mathrm{S}_{1}\right)+\mathrm{P}\left(\mathrm{S}_{2}\right)}{2}=\frac{\mathrm{S}_{1}+\mathrm{S}_{2}}{2}, \mathrm{P}\left(\mathrm{S}_{3}\right)=0.5$,

146

147

148

149

150

151

152

153

154

155

156

157

158

$$
\sum_{\mathrm{i}=1}^{\mathrm{n}} \mathrm{S}_{1}(\mathrm{i}) \mathrm{S}_{2}(\mathrm{i})=\frac{\sum_{\mathrm{i}=1}^{\mathrm{n}} \mathrm{S}_{1}(\mathrm{i}) \times \sum_{\mathrm{i}=1}^{\mathrm{n}} \mathrm{S}_{2}(\mathrm{i})}{\mathrm{n}},
$$

where $\mathrm{P}$ is the probability of the stochastic stream. The AND gate multiplier only applies to unipolar math where the real number $\in[0,1]$. In the case of bipolar math where real number $\in$ $[-1,1]$ ( 0 's bit decodes as -1$)$, the XNOR gate can be used as a multiplier, whereas the same MUX can function as a bipolar adder.

The stochastic number generator ( $\mathrm{SNG}$ ) becomes the heart of the SC to perform arithmetic operations in the stochastic domain. SNG consists of a random number generator (RNG) and a comparator; both worked synchronously to generate stochastic bitstream from a given binary number. However, the RNG was the biggest challenge in the previous SC circuit design because the correlation between the operating bitstreams plays a great role in SC accuracy. An SC output will be accurate only if both working streams are not correlated. Taking Fig. 2(C) and (D) as examples, $[0,1,1,0,1,0,0,1]$ and $[1,1,0,0,1,0,0,1]$ bitstreams can represent the value of $4 / 8$, but the output on Fig. 2(D) is far from accurate due to a high correlation to the opposite bitstream. The correlation index of both bitstreams can be defined as:
159

160

161

162

163

164

165

166

167

168

169

170

171

172

173

174

175

176

177

178

where ' $\mathrm{S}$ ' is the respective stochastic bitstream and ' $\mathrm{n}$ ' is the bit length. Thus, the accuracy is highly dependent on the randomness and the lengths of the stochastic stream. Nevertheless, not all of the SC elements are sensitive to stochastic correlation such as MUX scaled adder(Alaghi, Qian \& Hayes, 2018).

\section{Figure 2 here}

Presently, a pseudo-random RNG called a linear-feedback shift register (LFSR) is widely accepted due to its simple design and effectiveness in lowering bitstream correlation(Alaghi \& Hayes, 2013). LFSR consists of a feedback XOR gate and a bit shift register as shown in Fig. 3(A). The register will be initialised with a specific value, and then, the register will generate pseudo-random binary values in every bit shifting clock cycle. The binary number generated from RNG will be compared with the user input binary number. Two circuits can be used as a comparator, namely, binary comparator and weighted binary generator (WBG) as shown in Fig. 3(B) and (C) respectively. Both are capable of generating stochastic bitstream. After the stochastic stream passed through the stochastic logic circuits, the computed stochastic streams can be converted back to the binary domain by using a simple flip-flop counter.

Figure 3 here

PeerJ Comput. Sci. reviewing PDF | (CS-2020:07:51446:1:1:NEW 23 Sep 2020) 
179

180

181

182

183

184

185

186

187

188

189

190

191

192

193

194

195

196

197

198

199

200

201

202

203

204

205

206

207

208

209

210

211

212

213

214

215

SC never stops improving and keep achieving great accuracy whilst using less area and power. SNG is the major overhead of the SC circuit. As such, (Ichihara et al., 2014) proposed a circular shifting technique to share LFSR. Then, (Kim, Lee \& Choi, 2016a) proposed a method very similar to memoisation computing to reduce the number of LFSRs in large scale SC. (Xie et al., 2017) attempted to share LFSR with wire exchange technique with additional random bit flip, whereas (Joe \& Kim, 2019) proposed symmetrical exchange of odd wire and even wire. Even better, (Salehi, 2020) showed that simple wire permutation paired with WBG could deliver the lowest correlation index, thus achieving great accuracy whilst requiring fewer logic gates. Interestingly, (Chen, Ting \& Hayes, 2018) replaced LFSR with up-counter in conjunction with WBG to take advantage of WBG binary weighting characteristics to assure SC progressive precision behaviour. As such, zero-error EDT is achievable without extra hardware cost. The WBG could also be shared partially because some WBG logics could be redundant(Yang et al., 2018).

More advanced operations, such as square, division and non-linear functions, had also gained attention and innovations to fit modern applications. The stochastic square is already in its simplest form as shown in Fig. 4(A). Squaring stochastic stream can be conducted by delaying the input stream with D flip-flop before multiplying itself. In the case of a non-linear function, such as hyperbolic tangent (TanH), stochastic TanH (Stanh) uses k-state finite state machine (FSM), such as that in Fig. 4(B). FSM is a class of logic circuits that will have a specific logical output pattern only if the input reached a designated sequential threshold. Stanh function can be described as:

$\operatorname{Stanh}(K, x)=\tanh \left(\frac{K x}{2}\right)$,

where $\mathrm{K}$ is the number of states (must be multiples of 2) and $\mathrm{x}$ is the input stream. (Brown \& Card, 2001) showed that Stanh function responds closely to the true TanH function with $\mathrm{K}=16$. However, too many states will result in random walk behaviour(Kim et al., 2016); thus, an optimum amount of state for accurate reproduction of TanH function exists in the stochastic domain. An improvement in FSM design can also emulate linear and exponential functions (Najafi et al., 2017).

\section{Figure 4 here}

The real challenge in SC (also the missing part of SC) is the stochastic divider design. Stochastic division traditionally used FSM with extra SNG components for gradient descent approach as shown in Fig. 5(A) and (B), but the gradient descent convergence time incurred inaccuracy on the output. Newer SC divider from (Chen \& Hayes, 2016) exploited the stochastic correlation properties to perform stochastic division without using expensive SNG as shown in Fig. 5(C) and (D). This event is possible because if stochastic stream $p(x)$ and $p(y)$ are perfectly correlated, and $\mathrm{p}(\mathrm{x})<\mathrm{p}(\mathrm{y})$, then by probability math: 
$\mathrm{p}(\mathrm{x}=1, \mathrm{y}=1)=\mathrm{p}(\mathrm{x}=1)$.

216 Given that conditional probability $\mathrm{p}(\mathrm{x}=1 \mid \mathrm{y}=1)$ (probability of $\mathrm{x}=1$ given that $\mathrm{y}=1$ ) can also be

217 expressed as:

$$
\mathrm{p}(\mathrm{x}=1 \mid \mathrm{y}=1)=\frac{\mathrm{p}(\mathrm{x}=1, \mathrm{y}=1)}{\mathrm{p}(\mathrm{y}=1)}=\frac{\mathrm{p}(\mathrm{x})}{\mathrm{p}(\mathrm{y})},
$$

218 the desired divider function on the SC domain is derived as a result. Hence, the stochastic

219 division can be performed if both stochastic streams are perfectly correlated by evaluating the

220 conditional probability of $x$ and $y$. In the case of $p(x)>p(y)$, the output will be clipped to a value 221 of 1. (Chu, 2020) improved the circuit by using JK flip-flop, but only for unipolar SC division.

222

223

224

225

226

227

228

229

\section{Figure 5 here}

The overall structure of SC is thus explained. Other than the benefit of power efficiency, SC is also inherently error resilient where accidental bit flips will not affect the overall operation of the stochastic circuits; otherwise, it could be catastrophic in deterministic computing. Secondly, $\mathrm{SC}$ is inherently progressive precision whereby the output value converges from the most significant figures. For instance, if the output is 0.1234 , then ' $0.1 \ldots$ ' will show first in the

230

231 stochastic compute cycles instead of '...4' in the conventional binary. This characteristic is useful in specific applications, such as weather forecasting, where only the most significant

232

233 figure matters in decision making. Thus, performing EDT in SC without waiting for complete computation is possible. With that said, its simplicity did come at a cost. Increasing math precision in SC also requires long bit lengths, thus increasing computing time latency by $2^{n}$ folds. For instance, doubling numerical precision from 4 to 8 bits requires increasing bit length

236

237

238 from $2^{4}=16$ bits to $2^{8}=256$ bits, or $2^{4}$ times exponential increase in computing latency.

$\mathrm{SC}$ becomes unfavourable to modern computation needs due to the ever-increasing efficiency 239 in binary computing. Nevertheless, certain niche applications can still benefit from SC topology, such as a low-density parity-check decoder in a noisy data-transmission environment; very robust image processing tasks, such as gamma correction and Sobel edge detection (Joe \& Kim, 2019); and the recent interest in CNN algorithm.

242

243

\section{CNN}

244

With the advancement of computing technology, many applications are getting highly reliant on probabilistic computation. Deep neural network (DNN) is a widely accepted class of machine learning algorithms to process complex information, such as images and videos. The nature of DNN consists of layers of addition and multiplication of numerical weights that end up computing the overall dimensionless probability values of an output class, which in turn allows the computer to decide based on the output value. Many DNN algorithm variations exist, each for a particular purpose, such as $\mathrm{CNN}$ for image processing and long short-term memory for neural-linguistic processing. $\mathrm{CNN}$, for example, can reduce multidimensional images into simple 
252

253

254

255

256

$$
\tilde{\mathrm{x}}_{\mathrm{j}}^{1}=\mathrm{f}\left(\mathrm{x}_{\mathrm{j}}^{\mathrm{l}}\right)=\mathrm{f}\left(\sum_{\mathrm{i}=1}^{\mathrm{n}}\left(\mathrm{x}_{\mathrm{i}}^{1-1} \times \mathrm{w}_{\mathrm{ij}}^{1-1}\right)+\mathrm{b}_{\mathrm{j}}^{1}\right),
$$

257

258

259

260

261

262

263

264

265

266

267

268

269

270

271

272

273

274

275

276

277

278

279

280

281

282

283

284

285

286

classes; thus, $\mathrm{CNN}$ is very popular in image classification and object recognition.

The most distinctive component that discriminates CNN from other DNN algorithms is its convolution layer. CNN can reduce large matrices into a single value representation, as shown in Fig. 6(A), which explains its superior capability of dimensional reduction in image processing. The convolution process can be generalised as:

where $x_{j}^{1}$ is the convolved feature of the next layer, $x_{i}^{1-1}$ is the feature from the previous layer, $\mathrm{w}^{1-1}{ }_{\mathrm{ij}}^{1}$ is the kernel weight matrix, and $b_{j}^{1}$ is bias. ' 1 ' is the layer number, ' $\mathrm{i}$ ' denotes scan window number, ' $n$ ' is the total number of scan window, and ' $\mathrm{j}$ ' is the depth of next feature map. After the convolution, the activation function $\mathrm{f}\left(\mathrm{x}_{\mathrm{j}}^{1}\right)$ exists, which can be a linear or non-linear function. Rectified linear unit (ReLU) and Tanh are just the names of a few popular activation functions. The final product $\tilde{x}_{j}^{1}$ will be aggregated, and the process repeats, depending on the structure of the CNN model.

The convolution and activation layers are fundamental in CNN, albeit other optional layers exist, such as normalisation layer (to reduce output variance)(Ioffe \& Szegedy, 2015), pooling layer (to save memory), and dropout layer (to prevent overfitting)(Hinton et al., 2012). At the end of convolution, the convolved pixel matrix will be flattened into a single list of data. Then, those data will be fed to a highly traditional biological neuron-inspired model, so-called fully connected neuron or dense layer, as shown in Fig. 6(B). Moreover, multiplication and addition repeat until the model converges to the size of the desired class output. A simple LeNet-5 model(Liew et al., 2016) as depicted in Fig. 6(C) shows the end-to-end structure of a typical $\mathrm{CNN}$, from the input image to output class.

\section{Figure 6 here}

Its cascaded arithmetic operation is where the $\mathrm{CNN}$ algorithm execution stressed the modern processor. It either spends too much processor time to serialise the process, or taking many hardware resources for parallelisation. The convolution arithmetic does multiplication and addition exhaustively. If the matrices of scanning windows are large or the network is deep/wide, then the computational demands required are high. As the multiplication and accumulation operations increase, memory access bottlenecking becomes the major limitation for DNNs (Capra et al., 2020). Traditional computing also uses floating-point units (FPU) which takes a wide area with high power consumption due to the colossal amount of logic gates involved. As the edge computing gains interest as the future trends of computation, energy efficiency has become a major concern for the $\mathrm{CNN}$ development and urged the researchers to rethink another way to process the information efficiently. Most of the modern FPU is of 32-bit floating-point 
287 (full precision). Thus, reducing the precision to 16 bits (half precision) or lower is one of the

288

289

290

291

292

293

294

295

296

297

298

299

300

301

302

303

304

305

306

307

308

309

310

311

312

313

314

315

316

317

318

319

320

321

322

323

324

325

326

ways to improve CNN computation efficiency without much accuracy degradation.

\section{BNN}

In an extreme case, the parameters are reduced to only a single bit representation. This radical simplification of CNN is called BNN and gained attention among researchers in the industry due to its compactness in memory usage and practicality(Simons \& Lee, 2019). In BNN, the parameters can only have two possible values, that is, -1 or 1 . Despite some considerable degree of accuracy degradation, BNN does have several unique advantages. First is its model size; for instance, $64 \mathrm{MB}$ of parameter data can be reduced to $2 \mathrm{MB}$, thus allowing the deployment of small embedded systems. Its little memory usage also allows memory-centric computing where the parameters can be stored directly beside the processing elements, thereby speeding up the computation by eliminating data communication cost (Angizi \& Fan, 2017). Second is its hardware implementation capability. BNN requires some amount of arithmetic logic unit (ALU) to process fixed-point image data at the frontend (still cost less than FPU). However, the multiplication of the hidden layer can be simply an array of XNOR gates because the multiplication of -1 and 1 is of bipolar math. The high hardware utilisation of BNN in FPGA results in high throughput, whereas being an order of magnitude if not more energy-efficient than CPU and GPU despite lower clock speed(Nurvitadhi et al., 2017). Another unique advantage is that $\mathrm{BNN}$ is less susceptible to adversarial attack with stochastic weight quantisation in the hidden layer (Galloway, Taylor \& Moussa, 2018). The adversarial attack is where data, for example, an image, are injected with noise and adversely affect the output class decision of a fine-tuned CNN model, albeit the doctored image has no perceptual difference to human eyes.

However, non-linear functions become useless due to the extreme information loss by the parameter quantisation. Instead, a threshold function can simply replace the normalisation and activation functions(Simons \& Lee, 2019). The BNN also suffer accuracy degradation from highly challenging datasets, such as ImageNet classification, due to extreme information loss. As many studies explore for better BNN optimiser algorithms, (Zhu, Dong \& Su, 2019) found that training optimisers might not help much due to BNN insensitivity to small changes in value. Instead, BNNs in parallel with ensemble technique (multiple trained BNNs in parallel and final decision with a majority vote) is a perfect fit, improving the overall BNN accuracy on large image classification datasets.

\section{SC CNN vs BNN}

The evolution of CNN to BNN challenged the idea of SC due to the competitiveness in hardware implementation capability. SC implementation is technically more challenging than BNN due to various custom logics and substantial uncertainty in future community support. After all, SC is still at its infancy in the CNN domain. Regardless of the different intentions and directions of development of SC and BNN, both studies try to explore alternatives for a highly efficient computing paradigm in the future of the IoT edge computing. With the massive exploitation and 
327

328

329

330

331

332

333

334

335

336

337

338

339

340

341

342

343

344

345

346

347

348

349

350

351

352

353

354

355

356

357

358

359

360

361

362

363

364

365

366

integration of DNN algorithms into small or remote devices, such as a smartwatch or surveillance camera, both fields of studies will contribute to the development of a highly realistic edge computing ecosystem.

\section{SC implementation in CNNs}

SC is considered the next frontier in energy-efficient edge computing (Jayakumar et al., 2016) due to its energy-efficient operation and ability to tolerate errors in domains of recognition, vision, data mining and so on. Meanwhile, many applications attempt to offload challenging workloads from cloud computing to edge devices. Thus, SC had become the hotspot of research interest.

\section{Integral SC: a radical change in SC methodology for the sake of CNN}

$\mathrm{CNN}$ is very popular in vision application due to its simplicity and accuracy. However, SC does not provide out-of-box experience as SC is not yet customised and explicitly optimised for the CNN algorithm. Hence, (Ardakani et al., 2017) proposed a radical idea to use an integer stream instead of the traditional bitstream. The stochastic byte is $\in[0,1,2 \ldots]$ so that to repurpose simple binary multiplier and bitwise AND as shown in Fig. 7(B) and (C) to process integer number in the stochastic domain, or integral SC. The idea is to preserve information across different precisions within a limited stochastic length.

The effect of information loss becomes apparent when many MUX half-adding many stochastic streams exist together. The resultant precision requirements will only increase and require long overall bitstreams to preserve the precision of the half-added stochastic number. For example, a value of $0.5625(9 / 16)$ requires a 16-bit length stochastic stream, whereas $0.875(7 / 8)$ only requires 8-bit length. Although 0.875 can be expressed in 16-bit length, half-adding both numbers result in 0.71875 (23/32), or at least 32-bit length to preserve the output precision in the stochastic domain. If so, the overall stochastic bit length will need to be extended to 32-bit length. Cascading MUX adders in the CNN convolution stage will drive up the bit length requirements drastically, thus incurring considerable computational latency. The same problem also applies to the multiplier.

Then, the integral SC comes into play. Take Fig. 7(A) as an example. A value of 0.5625 can be effectively represented in the same length as the 8-bit length value of 0.875 . Given that integral SC can preserve the stochastic information in an integer value, the final batch adding operation in CNN can be processed with tree adder as shown in Fig. 7(D), eliminating parallel counter. Integer stream also allows short stochastic stream length, thus speeding up the SC time. They also proposed integer version of TanH k-state FSM because the traditional stochastic TanH (Stanh) function on FSM only accepts stochastic bits, thereby leading to the modern TanH FSM design. However, integral SC only solved the precision degradation issue, and many other CNN functions are yet to translate to SC domain. Moreover, the usage of binary adder and multiplier may not scale well in the case of deploying large CNN models. They claimed energy saving of $21 \%$ compared with the full binary radix computing but is still far from the expected power 
367

368

369

370

371

372

373

374

375

376

377

378

379

380

381

382

383

384

385

386

387

388

389

390

391

392

393

394

395

396

397

398

399

400

reduction in the SC transition.

\section{Figure 7 here}

\section{Extended stochastic logic (ESL): another radical approach}

ESL made an extreme modification to the SC methodology if integral SC is not radical enough. Instead of using a single stochastic bitstream for a value, ESL used two stochastic streams such that their ratio of division represents the actual value (Canals et al., 2016). ESL intends to compute the entire range of real numbers in the stochastic domain. For example, if $x^{*}$ is a whole number, then $\mathrm{x}^{*}=\mathrm{p}^{*} / \mathrm{q}^{*}$, where $\mathrm{p}^{*}$ and $\mathrm{q}^{*}$ are the ESL stochastic pair for $\mathrm{x}^{*} . \mathrm{p}^{*}$ and $\mathrm{q}^{*}$ remain in real number $\in[-1,1]$ in the bipolar format, but obtaining its ratio $x^{*}$ can translate to the entire range of real numbers $\in[-\propto, \propto]$.

ESL requires dedicated logic gate for $\mathrm{p}^{*}$ and $\mathrm{q}^{*}$ stochastic streams. Taking Fig. 8(A) and (B) as an example, if $\mathrm{x}^{*}=\mathrm{p}^{*} / \mathrm{q}^{*}$ and $\mathrm{y}^{*}=\mathrm{r}^{*} / \mathrm{s}^{*}$, then by probability math, multiplication between two separable stochastic streams will be:

$$
\mathrm{x}^{*} \times \mathrm{y}^{*}=\frac{\mathrm{p}^{*} \times \mathrm{r}^{*}}{\mathrm{q}^{*} \times \mathrm{s}^{*}}=\frac{\mathrm{t}^{*}}{\mathrm{u}^{*}},
$$

where $t^{*}$ and $u^{*}$ are the output pair of stochastic streams. Division can be done simply by flipping the nominator and denominator of the second stochastic pair. In the case of stochastic addition, the stochastic pair can be processed such that:

$$
\mathrm{x}^{*}+\mathrm{y}^{*}=\frac{\mathrm{p}^{*}}{\mathrm{q}^{*}}+\frac{\mathrm{r}^{*}}{\mathrm{~s}^{*}}=\frac{\mathrm{p}^{*} \times \mathrm{s}^{*}+\mathrm{q}^{*} \times \mathrm{r}^{*}}{\mathrm{q}^{*} \times \mathrm{s}^{*}}=\frac{\mathrm{t}^{*}}{\mathrm{u}^{*}},
$$

whereas subtraction can be done by NOT gate inversion as shown in Fig. $8(\mathrm{C})$.

\section{Figure 8 here}

Value splitting is feasible in the stochastic domain due to the nature of probabilistic computing. However, splitting into double stochastic streams complicated everything, including a compulsory custom bipolar divider (convert $t^{*}$ and $u^{*}$ back to real number representation) before bipolar TanH function blocks. The custom block extensively used comparator and RNG, which add a red flag for efficient computing. The neural network may compute in the real number $\in[-\propto, \propto]$ on the early day, but the CNN nowadays commonly compute in bipolar math. After all, the final output class of $\mathrm{CNN}$ only need to tell the computer whether the probability $\epsilon$ $[0,1]$. ESL did provide an insight into how SC can perform normal arithmetic full-range computation. However, verifying whether ESL is better than other SC methods for CNN use case despite the attractive circuit simplicity in primary arithmetic operations is hard due to the nonlinear activation function complexity in ESL implementation. 
401 Approximate parallel counter (APC) and Btanh: a simple and energy-efficient approach

402 Implementing radical changes in every SC component might not be easy. Thus, another highly

403 effective approach with traditional stochastic bitstream is APC. Other than the frontend binary to

404 stochastic conversion stage of SNGs, the final stochastic to binary conversion stage is also

405 equally important (Kim et al., 2016 a; Kim, Lee \& Choi, 2016b b).

406 In the case of accumulating multiple bitstreams, MUX adder could become inaccurate due to 407 loss of $n-1$ bits input information( $\mathrm{Li}$ et al., 2017c). In this case, a parallel counter as the one in

408 Fig. 9(B) is preferred consisting of an array of full adders (FA), but FA is relatively expensive as 409 it uses binary adder logic circuits. The accurate parallel counter should no longer be used as SC

410 is already based on approximate computation. Thus, an APC has been proposed to reduce the FA 411 components with a slight trade-off on accuracy whilst achieving the same counting function at 412 lower area and power consumption as shown in Fig. 9(A). The proposed APC could save area 413 and power by $38.3 \%$ and $49.4 \%$, respectively. The caveat is that the output from APC is in the 414 binary domain; thus, directly removing any stochastic stream from the stochastic domain 415 computation.

416 Although the traditional Stanh uses single input k-state FSM, with the inspiration from 417 integral SC research, the binary output from APC is cleverly reused as an input for another 418 modified binary input FSM called Btanh. TanH activation function is essential in CNN. For 419 example, if the binary output value is 4, then the FSM will directly jump four states instead of

420

421

422

423

424

425

426

427

428

429

430

431

432

433

434

435

436

437

438

439

440 step-wise jumps in Stanh. More granular Tanh step-function could also be achieved, which is not possible with Stanh FSM. In the end, the binary output values from APC will be indirectly converted back to stochastic stream with TanH non-linear function applied, completing the stochastic convolution computation as depicted in Fig. 9(C). Moreover, energy usage can be further reduced by $69 \%$ by sacrificing $1.53 \%$ of accuracy with EDT, that is, terminating computation at $50 \%$ of the computing time. Then, their APC and Btanh components had become the foundation for other SC CNN approaches in the next coming years.

Figure 9 here

\section{Near-perfect SC implementation in CNN algorithm}

(Ren et al., 2016; Li et al., 2017c,b; Ren et al., 2017; Li et al., 2018a) proposed a complete overview of a near-perfect CNN analogy in the SC domain, including the following: the missing pooling layer, ReLU and sigmoid activation layer, and normalisation layer which will be discussed separately in the sub-sections below.

\section{$\underline{\text { SC average pooling and max-pooling layers }}$}

The purpose of CNN pooling layer is to reduce memory usage and reduce model size. (Ren et al., 2016) first used cascaded MUX as the average pooling function in CNN as shown in Fig. 10(A). This solution is simple but may face the precision loss issue as described in the Integral SC research. Average pooling may not help in CNN training convergence either. (Ren et al., 2017) 
441 proposed max-pooling hardware equivalent to the widely adopted CNN max-pooling layer. The 442 stochastic stream with a maximum value at any given time in the stochastic domain could not be

443 verified. Hence, a dedicated counter for each stochastic stream is required to sample and evaluate 444 which stream is of maximum value. By referring to Fig. 10(B), the counter samples the first few 445 bits and compare the magnitude at the end of bitstream sampling to make an early decision on 446 which stochastic stream should be chosen to continue the path. The first few bit information 447 could be inaccurate and thus is an approximate max pooling. Nevertheless, the decision will 448 eventually converge to the bitstream of maximum value if the sampling continues due to the 449 properties of SC progressive precision. Moreover, if the bitstream is long, then less information 450 will be lost, thereby achieving negligible accuracy loss.

451

452

453

454

455

456

457

458

459

460

461

462

463

464

465

466

467

468

469

470

471

472

473

474

475

476

477

478

479

\section{Figure 10 here}

$$
\mathrm{f}(\mathrm{x})=\max (0, \mathrm{x}) \text {. }
$$

However, a more straightforward stochastic max-pooling block was proposed by (Yu et al., 2017). With only an XOR gate, FSM and MUX, a novel stochastic MAX block could select whichever stream of higher value. With XOR gate controlling the FSM state jumping, the probability of the opposite stream could be inferred from another bitstream by generating the condition of bit entanglement. As such, whenever the FSM sampled a 0's bit from the current bitstream, it implies a 1's bit on the opposite bitstream. Thus, whenever inequality between two bitstreams exists, the FSM state will be biased to the one with higher magnitude, completing the MAX function with the MUX. Cascading the MAX function block could realise the max-pooling function block as shown in Fig. 10(C).

\section{$\underline{\text { SC ReLU and Sigmoid activation layer }}$}

The CNN activation layer is similar to the usual neuron activation function. ReLU function, as the name suggests, performs rectification and cutting off any negative value such that:

ReLU function is trendy due to its fast computation and solves diminishing return in backward propagation learning during the $\mathrm{CNN}$ training stage. However, no $\mathrm{SC}$ equivalent circuit existed for that particular function; thus, (Li et al., 2018a) proposed a novel SC-based ReLU function block as depicted in Fig. 11(A).

Firstly, the ReLU amplitude will be naturally maxed out at value $=1$ in the stochastic domain, but this is not a concern as clipped ReLU has no significant accuracy degradation (FeiFei, Deng \& Li, 2010). Secondly, a negative value must be clipped to zero. Notably, the number of 0 's bit in the bipolar stochastic stream determines the magnitude of negativity. Thus, when the accumulated value is less than the reference half value (the 0's bit is more than 1's bit) in a given sample time, the output will be forced to be 1's bit. Otherwise, the output will follow the pattern of emulated linear function from the FSM. Although real number convergence in the accumulator takes time, the real value information is equally distributed in the stochastic bitstream. Hence, obtaining an accurate comparison is possible by observing the first few bits of information; thus, inaccuracy is negligible. Moreover, the comparison is synchronous to the

PeerJ Comput. Sci. reviewing PDF | (CS-2020:07:51446:1:1:NEW 23 Sep 2020) 
480

481

482

483

484

485

486

$$
\mathrm{A}=\frac{1}{4} * \sum_{\text {pos }} \mathrm{P} \cdot \mathrm{Q}+\frac{1}{2}+\frac{\text { bias }^{+}}{4}, \mathrm{~B}=\frac{1}{4} * \sum_{\mathrm{neg}} \mathrm{P} \cdot \mathrm{Q}+\frac{\text { bias }^{-}}{4}
$$

487 488

$$
\mathrm{A}-\mathrm{B}=\frac{1}{2}+\frac{1}{4}\left(\sum \mathrm{P} \cdot \mathrm{Q}+\text { bias }\right)
$$

489

input; therefore, no latency will be incurred. function becomes more favourable as non-linear function. As such, (Li et al., 2017a) proposed a hardware-oriented SC sigmoid approximation function as shown in Fig. 11(B). Since the output of the stochastic stream is maxed at 1 , the Taylor series expanded sigmoid function could be approximated as:

$\frac{1}{1+\exp (-\mathrm{x})} \approx\left\{\begin{array}{c}1, \quad \mathrm{x}>2 \\ \frac{1}{2}+\frac{1}{4} \mathrm{x}, \quad-2 \leq \mathrm{x} \leq 2 . \\ 0, \mathrm{x}<-2\end{array}\right.$

where ' $\mathrm{P}$ ' and ' $\mathrm{Q}$ ' are the weight and pixel value respectively. Therefore, by pre-scaling the weights and bias to quarter times, the stochastic sigmoid function could be devised as a result, with the added benefit of including bias information which is missing in the previous SC CNN implementation. The binary adder now is the sigmoid activation function itself, eliminating the need for extra hardware cost such as FSM. However, unlike the APC + Btanh function block, the accurate parallel counter is needed.

The sigmoid function is not limited to $\mathrm{CNN}$ algorithm, or rather, is a universal activation function in other DNN classifier algorithms such as multilayer perceptron and restricted Boltzmann machine. With 1024-bit length stochastic stream, the proposed SC sigmoid activated convolution neuron block could perform as accurate as binary computing CNN while consuming $96.8 \%$ and $96.7 \%$ less area and power respectively, hugely improving the capability of SC in the DNN algorithm computation in general.

\section{Figure 11 here}

$\underline{\text { SC normalisation layer }}$

The purpose of the normalisation layer is to reduce internal covariance, thereby improving the overall CNN output accuracy. If the ReLU activation is applied to the previous layer, only a simple local response normalisation function is required, which can be summarised as: 


$$
b_{x, y}^{i}=\frac{a_{x, y}^{i}}{\left(k+\alpha \sum_{j=\max (0, i-n / 2)}^{\min (N-1, i+n / 2)}\left(a_{x, y}^{j}\right)^{2}\right)_{\beta}^{\beta}},
$$

508 where the summation part accumulates all $\mathrm{N}$ numbers of adjacent neuron output of $\mathrm{a}_{\mathrm{x}, \mathrm{y}}^{\mathrm{i}}$. ' $\mathrm{k}$ ', ' $\mathrm{n}$ ',

509 ' $\alpha$ ', and ' $\beta$ ' are hyperparameters which can be determined by CNN backpropagation training.

510 The complexity of the mathematical relationship can be decoupled into three compute

511 components, square and sum (calculate the denominator components), exponential function with

512 " $\beta "$ and finally division. (Li et al., 2017c) used stochastic square, FSM activation block and

513 traditional gradient descent SC divider to construct SC normalisation circuit as shown in Fig. 12

514 to perform SC normalisation. The accuracy had improved with SC normalisation function and

515 only dropped by $0.88 \%$ compared with the original binary AlexNet CNN model, achieving six

516 times in the area and five times in power savings compared with binary equivalent normalisation.

517 However, they could have utilised newer SC divider as discussed in the basic concept section

518

519

Figure 12 here

520

521

\section{Other optimisations}

522

The dropout layer is one of the regularisers in CNN to prevent overfitting. However, dropout

523 layer functions only at the CNN training phase, and no custom hardware adaptation is needed at

524 the inference stage, hence no extra hardware overhead. (Li et al., 2018a) optimised the APC

525 function block by utilising inverse mirror FA to reduce the number of transistors required for single FA from 32 to 24 transistors. They also proposed the APC design which input is not a power of two by incorporating inverse half adder. APC optimisation further reduced the area required by at least $50 \%$ and an average of $10 \%$ improvement in energy efficiency.

529

In terms of SC accuracy, the bipolar format remains the major limitation as bipolar is generally worse than the unipolar in terms of SC accuracy(Ren et al., 2016). To overcome the

531 signed value accuracy limitation, (Zhakatayev et al., 2018) decoupled the sign information from

532 the stochastic stream and added one stochastic bit pair specifically to store the sign value. Unlike stochastic probability value, the sign value of a stochastic stream is deterministic, thus, can be processed separately from the stochastic magnitude. Although small hardware overhead is needed to process the sign function, such as an extra XOR gate to multiply signed value, the accuracy gain is significant, 4 9.5 times better compared to the bipolar format. With that advantage in mind, the little extra hardware cost for sign processing is trivial.

538

\section{Binary Interlaced SC, two is better than one}

540 Full-fledged SC CNN might not be feasible to fit a wide variety of modern complex CNN

541 models. However, the massive multiplication parallelism of SC is still very favourable. Thus SC-

542 based multiply-accumulate (MAC) unit was proposed by (Sim et al., 2017) as shown in Fig. 
543 13(A) to act as multiplier accelerator for binary computing. The MAC leverages the parallelism

544 of SC multiplier, then accumulate value with accurate parallel counter, returning pure binary

545 value to other binary computing circuits at the end of SC cycle. This approach, while not the

546 most energy-efficient one, achieved two times the area efficiency and at very high throughput

547 compared to binary computing. With only a single layer SC in mind, (Sim \& Lee, 2017) further

548 leveraged the SC MAC to perform unipolar SC multiplication. All the stochastic 1's bit of the

549 neuron weight value was pushed ahead of time by down counting the weight value so that the SC

550 cycle could terminate when the stream tail of the weight ended with 0 's bit as depicted in Fig.

551 13(B). This event is possible because any section of the stream could represent the true value of

552 the stream due to the probabilistic nature. It is technically feasible as long as single layer SC is

553 concerned. They also proposed a novel MUX FSM based SNG. By predefining the MUX

554 selection sequence in such a way that the output is the sum of binary weight, the binary input

555

556

557

558

559

560

561

562

563

564

565

566

567

568

569

570

571

572

573

574

575

576

577

578

579

580

581

582 could be directly converted into a stochastic stream as depicted in Fig. 13(C), eliminating the need of WBGs which could be expensive in FPGA implementation. With the strategic downcounting timing, an area-delay product reduction of $29 \% \sim 49 \%$ is achieved while being $10 \% \sim 29 \%$ more energy efficient compare to binary computing. In any case, they ignored the SNGs hardware overhead in performance comparison.

\section{Figure 13 here}

Considering that only a single SC layer is required, (Hojabr et al., 2019) radically redesigned the MAC unit by exploiting computing pattern in modern CNN design and proposed Differential MAC (or DMAC). Firstly, because CNN ReLU function always returns positive value, in addition to the binary pixel of positive value, thus, up/down counter could be used as ReLU function. Secondly, considering that a pixel value will eventually pass through all the weight multiplication matrix of CNN scanning window in the convolution process, the neuron weights could be sorted offline ahead of time. In this way, the weight differential from the next sorted weight of higher value is guaranteed to be positive, thus, can be fed to a down counter similar to SC MAC to pipeline the stochastic multiplication. Since the first weight is of minimum value which could be negative, a D Flip-Flop is used to hold the sign information just for the first bipolar multiplication. Thus, multiplying in $\mathrm{SC}$ is as simple as counting the number of bits from the MUX AND-ing with counter 'enable' control from the weights as depicted in Fig. 14. The FSM could be shared among all MUX, ignoring the stochastic correlation issue because the multiplication is mutually independent(Yang et al., 2018). The buffered accumulated value will then continue the summation operation as the DMAC final stage. This major circuit overhauling could deliver 1.2 times and 2.7 times gains in speed and energy efficiency respectively relative to the former MAC with the benchmarking on more modern CNN models.

\section{Figure 14 here}




\section{Stochastic Quantisation, SC is going asynchronous}

584 In the face of quantised binary $\mathrm{CNN}$ whereby the arithmetic is lower than 8-bit precision, no

585 optimisation had been done on the SC CNN counterpart. SC could consume a lot of logics as 586 well, especially in CNN use case. Thus, (Li et al., 2018b) proposed a novel multiplier with 587 shifted unary code (SUC) adder. From the binary interlaced SC research, the weights do not have 588 to follow probability distribution as the pixel value does, as long as the next SC component is not 589 computing in the stochastic domain. By strategically using the weight information as a timing 590 control for SC multiplication, meaningful bits from each stream could be quantised and unified 591 into a single multiply-sum-averaged stochastic stream by OR-ing the parallel bitstreams 592 asynchronously as depicted in Fig. 15. The SUC adder significantly reduced the requirement of 593 parallel counter whereby its internal FA is expensive in the perspective of SC. The area and 594 power savings are significant as a result, as much as $45.7 \%$ and $77.9 \%$ respectively relative to

595

596

597

598

599

600

601

602

603

604

605

606

607

608

609

610

611

612

613

614

615

616

617

618

619

620

621

622 usual unipolar SC with less than 1\% accuracy loss compared to quantised binary CNN, paving the way for more efficient parallel counting accumulation mechanism in SC CNN.

\section{Figure 15 here}

\section{Analog-to-Stochastic Converter, SC CNN is ready to be embedded}

In the case of direct interfacing with analogue input, such as analogue camera sensor, Analog-toDigital Converter (ADC) is usually being deployed, but at the cost of requiring memory storage. (Zhang et al., 2019) proposed a novel converter, namely, Analog-to-Stochastic converter (ASC) as shown in Fig. 16(A) where the analogue voltage differential could be directly decoded into stochastic streams with thermometer encoding scheme. The stochastic stream could either be encoded via LFSR, counter, or newly proposed thermometer coding as depicted in Fig. 16(B). The thermometer coding is capable of generating parallel bit streams at once but has higher error compared to the others. Nevertheless, with long enough bitstream length, those error is negligible. The thermometer encoding enabled the design of novel ASC which allows SC CNN to be directly interfaced with analogue voltage input, eliminating ADC and memory storage.

\section{Figure 16 here}

\section{SC CNN is meant for memory-centric computing}

Notably, SC CNN does require a tremendous amount of weight data similar to fixed point binary CNN. Despite many SC CNN architecture innovations, however, without efficient weight storage near to SC elements, SC CNN will suffer memory bandwidth bottlenecking similar to the binary computing. Since the weight information is fixed from the training process, those data can be stored in a more area and power-efficient non-volatile Domain-Wall Memory (DWM) (Ma et al., 2018) built beside the SC elements. This strategy could eliminate memory bandwidth bottlenecking by bringing memory closer to the computing element, namely, memory-centric computing or in-memory computing. SC CNN can greatly benefit from memory-centric 
623 architecture due to the nature of massive parallelism. Memoisation approach could also be 624 executed in memory-centric design by storing the weight data directly in a predefined stochastic 625 bitstream representation instead of original binary values. As such, sequential read of stochastic 626 bit from DWM could use less energy while reducing the SNGs usage. Further area reduction 627 could be achieved by sharing APC and weights. Thus, an area and power reduction of $52.6 \%$ and 62817.35 times were reported respectively relative to standard $\mathrm{SC} C N N$ as a result of resource 629 sharing and more efficient memory-centric architecture in the SC CNN circuit.

630

\section{SC implementation in BNN: the best of both worlds}

632 As mentioned earlier in the basic concept section, BNN challenged the existence of SC circuits in $\mathrm{CNN}$ computing. As the saying goes, the enemy of an enemy is a friend, and considering that $\mathrm{SC}$ and BNN target efficient CNN computation, why not combine both to maximise the benefits from both aspects, which is what (Hirtzlin et al., 2019) precisely targeted for. The inspiration for this particular approach is that the SC and BNN come into the same conclusion that XNOR gate can be used as a bipolar multiplier, as depicted in Fig. 17(A), despite different directions of development. If somehow a way to process the BNN model in stochastic mean exists, then the $\mathrm{SC}$ can take a free ride to the BNN's internal logic.

Although BNN process information at the bitwise level in the hidden layer, the initial layer still needs to deal with input images of fixed-point binary number as shown in Fig 17(B). In most cases, ALU is utilised for real number calculation, or digital signal processing unit in the case of FPGA. They attempted to fuse the SC domain onto the first layer by translating image input into stochastic bitstreams and then exploiting SC logic similarity in BNN for bipolar multiplication to take advantage of the BNN logic. However, unique data pre-processing is needed so that the trained network is trained on a serialised stochastic binary image instead of the original grayscale image. The input image is converted into multiple stochastic image representations as shown in Fig. 17(C) where the bitstream generation of each pixel follows the function of SNG. Then, the number of stochastic images generated is equal to the stochastic bit length of the data. A 'popcount' accumulator is implemented at the end of the layer to restore the real number before proceeding to the next threshold function, which had replaced the activation function and batch normalisation. The difference of their BNN usage compared with the general BNN is that they treated the BNN XNOR gate as if it is of SC CNN stochastic logic. Notably, the SC only apply

655 on the first layer, and the rest of the hidden layer still follows BNN logics.

657

\section{Figure 17 here}

In the end, they claimed to have $62 \%$ area reduction whilst only suffer $1.4 \%$ accuracy degradation in Fashion-MNIST dataset classification compared with the binary first-layer BNN. They also claimed that with three stochastic image representations, SC BNN could achieve the same performance as binary BNN implementation at 2.4 times lower energy usage, which is very similar to the EDT approach. They even extended the experiment with advanced CIFAR-10 
663 images with RGB channels. By following the same image conversion principle in channel-wise, 664 the SC BNN achieved the same accuracy as full binary BNN, proving that eliminating ALU at 665 the first BNN layer is possible. Nevertheless, one possible confusion is that they could have 666 mistaken the BNN weight information as part of the stochastic domain. The BNN weights were 667 trained in the binary domain with images of real fixed-point value, but it is not a concern as long 668 as the BNN weights are represented in fully quantised ' -1 ' or ' 1 ' vector regardless of the 669 computing domain.

670

\section{Discussion}

672 We discussed the SC CNN and BNN elements in component-wise. However, a visualisation

673 approach is necessary to obtain the full picture of how are they exactly being stacked together as 674 SC CNN and SC BNN, which no one had emphasised on in almost all related studies. Otherwise, 675 novel readers might be having a hard time to grasp the idea and motives behind the effort of SC 676 development, particularly for those studies mentioned above with the mixed bag of vastly 677 different fields of study.

678

\section{SC CNN and SC BNN from a holistic perspective}

680

681 Modern computing handles the $\mathrm{CNN}$ computation by aggregating all values layer-by-layer until

682

683

684

685

686

687

688

689

690

691

692

693

694

695

696

697

698

699

700

701

702 the final class output is converged. The hidden truth behind the oversimplified drawing of CNN as in Fig. 6(C) is that there could have a lot of data accumulation and transfer between the processor and memory. Even if modern GPUs could parallelise thousands of arithmetic operations, it still takes time to buffer computed data into local memory for each feature map or layer, because it is impossible to read and write on the same memory at the same time.

Conversely, SC handles the computation information in a different approach as depicted in Fig.18. Due to the extreme parallelisation capability of the SC circuit, all of the data could be technically preloaded into local memory before the starting of the SC cycle. Although stochastic stream could take hundreds or even thousands of clock cycles to complete (each clock for each stochastic bit), SC pipelined all CNN arithmetic operation from top-down. Thus, all of the bits at a particular moment passed though all CNN layers at every SC clock cycle. If a clock cycle took $1 \mu \mathrm{s}$, then a full-fledged SC CNN inference with 1-kilobit length stochastic streams could, in theory, complete the CNN computation in under $1 \mathrm{~ms}$. By then, a new full-sized image data could have been buffered asynchronously readily available for the next $\mathrm{SC}$ cycle. Thus, in the perspective of the SC circuit, memory bandwidth bottlenecking might not be an issue. The simple computing elements in SC allow large-scale parallelisation, which is incredibly favourable to $\mathrm{CNN}$ hardware implementation in edge computing application. The advantage will only be highly prevalent when noise tolerance is essential at a higher clock speed in the future of computing or deployment of a big $\mathrm{CNN}$ model which requires larger data parallelisation.

\section{Figure 18 here}


703

704

705

706

707

708

709

710

711

712

713

714

715

716

717

718

719

720

721

722

723

724

725

726

727

728

729

730

731

732

733

734

735

736

737

738

739

740

741

742

In the case of SC BNN as illustrated in Fig. 19, the converted stochastic images could exploit the BNN XNOR logic for SC, eliminating the need for ALU. Although the SC domain ended at the first layer, the subsequent BNN bipolar multiplication, accumulation and threshold loops do not take much computing time either, virtually single-layer pass in one or few clock cycles. Given the nature of the layer-wise operation, BNN could in practice allow layer folding, that is, reusing the computer components of the previous layer by reloading weight information (Mittal, 2020), further reducing the area and power required which are not possible on SC CNN. SC BNN also allows in-memory computation because those bit weights can be stored right next to the computing gate arrays, further improving energy efficiency by eliminating the cost of communication bandwidth. The ensemble technique on BNN could also perform as accurate as full precision DNN (Zhu, Dong \& Su, 2019). Thus, the area and power savings of SC BNN could be extreme, challenging the performance of SC CNN.

\section{Figure 19 here}

Although no standard reference exists for a fair comparison, we can compare the performance difference of SC CNN/BNN in CNN model-wise as shown in Table 1 to highlight the clear advantage of SC in CNN application. Nevertheless, the year of comparable studies varies greatly, and hardware and software efficiencies had greatly improved over the last decade, thus should only be taken as a rough comparison. In the case of component-wise performance comparison, Table 2 could further clarify the performance number that had been mentioned in the previous section if any.

\section{Table 1 here}

\section{Table 2 here}

\section{Conclusions}

The SC may still not well developed relatively speaking. Still, with the trending of highly parallelised computing use case, SC might be the good old yet not-so-old idea, specifically when people are still actively researching and optimising SC circuits with the driving momentum of CNN algorithm. That being said, the FPGA itself is still not widely adopted in the programming community, let alone the SC adaptation. Numerous efforts were made in the high-level CNN to FPGA translation for binary domain computation (Liu et al., 2017; Noronha, Salehpour \& Wilton, 2019). However, the bridging effort of SC in FPGA is near to non-existence or should be said most of the SC studies lean to ASIC. Many people are interested in offloading computationally intensive workloads such as image processing and CNN inferencing to the coprocessor. Thus, SC elements should be made an open-source IPs and introduced into the FPGA design ecosystem so that people can innovate on it. The open-sourcing design could help accelerate the SC development because researchers do not have to redesign the IP from scratch 
743 which is the major hurdle for novel development and could turn down people from being

744 interested in SC technology. It could be the primary reason why SC CNN lacks attention, leading

745 to a low number of comparable data as well as benchmarking.

746 Speaking of parallelism capability in SC, data bandwidth bottlenecking could be a major 747 challenge. Even though SC can have vast arrays of WBG or comparator to compare a massive

748 amount of binary values at once, delivering massive data on time is challenging. Notably, SC

749 does require hundreds if not thousands of clock cycles to complete. Thus, data transfer could be

750 pipelined and buffered asynchronously. Moreover, a tremendous amount of data needs to be

751

752

753

754

755

756

757

758

759

760

761

762

763

764

765

766

767

768

769

770

771

772

773

774

775

776

777

778

779

780

781

782

783

784

785 ready beside the SC elements. As such, local memory element such as SRAM (in ASIC terms) or BRAM/Flip-flop (in FPGA term) limitation should be the concern. In any case, memory-centric computing design should be the direction of SC development, especially in SC CNN, where hundreds of thousands, even millions of operations could be parallelised.

There are still a lot of optimisation rooms for SC implementation on FPGA since most of the modern FPGA consists of 6-input lookup tables. ASIC logic might not be able to translate into the FPGA fabric efficiently because lookup tables are hardwired. Although FPGA is flexible in terms of hardware implementation, it is not as customisable as the ASIC. Modern FPGA also consists of other resources capable of performance computing such as digital signal processors or arithmetic logic awaiting to be utilised. However, those aspects could only be discovered in future research efforts.

\section{References}

De Aguiar JM, Khatri SP. 2015. Exploring the Viability of Stochastic Computing. Proceedings of the 33rd IEEE International Conference on Computer Design, ICCD 2015:391-394. DOI: 10.1109/ICCD.2015.7357131.

Alaghi A, Hayes JP. 2013. Survey of Stochastic Computing. Transactions on Embedded Computing Systems 12. DOI: 10.1145/2465787.2465794.

Alaghi A, Qian W, Hayes JP. 2018. The Promise and Challenge of Stochastic Computing. IEEE Transactions on Computer-Aided Design of Integrated Circuits and Systems 37:1515-1531. DOI: 10.1109/TCAD.2017.2778107.

Angizi S, Fan D. 2017. IMC: Energy-Efficient In-Memory Convolver for Accelerating Binarized Deep Neural Network. ACM International Conference Proceeding Series 2017-July. DOI: $10.1145 / 3183584.3183613$.

Ardakani A, Leduc-Primeau F, Onizawa N, Hanyu T, Gross WJ. 2017. VLSI Implementation of Deep Neural Network Using Integral Stochastic Computing. IEEE Transactions on Very Large Scale Integration (VLSI) Systems 25:2688-2699. DOI:

10.1109/TVLSI.2017.2654298.

Brown BD, Card HC. 2001. Stochastic Neural Computation I: Computational Elements. IEEE Transactions on Computers 50:891-905. DOI: 10.1109/12.954505.

Canals V, Morro A, Oliver A, Alomar ML, Rosselló JL. 2016. A New Stochastic Computing Methodology for Efficient Neural Network Implementation. IEEE Transactions on Neural Networks and Learning Systems 27:551-564. DOI: 10.1109/TNNLS.2015.2413754.

Capra M, Bussolino B, Marchisio A, Shafique M, Masera G, Martina M. 2020. An Updated Survey of Efficient Hardware Architectures for Accelerating Deep Convolutional Neural 
786

787

788

789

790

791

792

793

794

795

796

797

798

799

800

801

802

803

804

805

806

807

808

809

810

811

812

813

814

815

816

817

818

819

820

821

822

823

824

825

826

827

828

829

830

831

Networks. Future Internet 12:113. DOI: 10.3390/fi12070113.

Chen TH, Hayes JP. 2016. Design of Division Circuits for Stochastic Computing. Proceedings of IEEE Computer Society Annual Symposium on VLSI, ISVLSI 2016-Septe:116-121. DOI: 10.1109/ISVLSI.2016.48.

Chen TH, Ting P, Hayes JP. 2018. Achieving Progressive Precision In Stochastic Computing. 2017 IEEE Global Conference on Signal and Information Processing, GlobalSIP 2017:1320-1324. DOI: 10.1109/GlobalSIP.2017.8309175.

Chu SI. 2020. New Divider Design for Stochastic Computing. IEEE Transactions on Circuits and Systems II: Express Briefs 67:147-151. DOI: 10.1109/TCSII.2019.2906385.

Fei-Fei L, Deng J, Li K. 2010. ImageNet: A Large-Scale Hierachical Image Database. Journal of Vision 9:1037-1037. DOI: 10.1167/9.8.1037.

Galloway A, Taylor GW, Moussa M. 2018. Attacking Binarized Neural Networks. 6th International Conference on Learning Representations, ICLR 2018 - Conference Track Proceedings:1-14.

Hinton GE, Srivastava N, Krizhevsky A, Sutskever I, Salakhutdinov RR. 2012. Improving neural networks by preventing co-adaptation of feature detectors. ArXiv e-prints:1-18.

Hirtzlin T, Penkovsky B, Bocquet M, Klein JO, Portal JM, Querlioz D. 2019. Stochastic Computing for Hardware Implementation of Binarized Neural Networks. IEEE Access 7:76394-76403. DOI: 10.1109/ACCESS.2019.2921104.

Hojabr R, Givaki K, Tayaranian SMR, Esfahanian P, Khonsari A, Rahmati D, Najafi MH. 2019. SkippyNN: An Embedded Stochastic-Computing Accelerator for Convolutional Neural Networks. Proceedings - Design Automation Conference:1-6. DOI: 10.1145/3316781.3317911.

Ichihara H, Ishii S, Sunamori D, Iwagaki T, Inoue T. 2014. Compact and Accurate Stochastic Circuits with Shared Random Number Sources. 2014 32nd IEEE International Conference on Computer Design, ICCD 2014:361-366. DOI: 10.1109/ICCD.2014.6974706.

Ioffe S, Szegedy C. 2015. Batch Normalization: Accelerating Deep Network Training by Reducing Internal Covariate Shift. Proceedings of the 32nd International Conference on Machine Learning 37:730-743. DOI: 10.1080/17512786.2015.1058180.

Jayakumar H, Raha A, Kim Y, Sutar S, Lee WS, Raghunathan V. 2016. Energy-Efficient System Design for IoT Devices. Proceedings of the Asia and South Pacific Design Automation Conference, ASP-DAC 25-28-Janu:298-301. DOI: 10.1109/ASPDAC.2016.7428027.

Joe H, Kim Y. 2019. Novel Stochastic Computing for Energy-Efficient Image Processors. Electronics (Switzerland) 8:1-11. DOI: 10.3390/electronics8060720.

Kim K, Kim J, Yu J, Seo J, Lee J, Choi K. 2016. Dynamic Energy-Accuracy Trade-off Using Stochastic Computing in Deep Neural Networks. Proceedings - Design Automation Conference 05-09-June. DOI: 10.1145/2897937.2898011.

Kim K, Lee J, Choi K. 2016a. An Energy-Efficient Random Number Generator for Stochastic Circuits. Proceedings of the Asia and South Pacific Design Automation Conference, ASP$D A C$ 25-28-Janu:256-261. DOI: 10.1109/ASPDAC.2016.7428020.

Kim K, Lee J, Choi K. 2016b. Approximate De-randomizer for Stochastic Circuits. ISOCC 2015 - International SoC Design Conference: SoC for Internet of Everything (IoE):123-124. DOI: 10.1109/ISOCC.2015.7401667.

Li Z, Li J, Ren A, Cai R, Ding C, Qian X, Draper J, Yuan B, Tang J, Qiu Q, Wang Y. 2018 a. HEIF: Highly Efficient Stochastic Computing-Based Inference Framework for Deep Neural Networks. IEEE Transactions on Computer-Aided Design of Integrated Circuits and 
832

833

834

835

836

837

838

839

840

841

842

843

844

845

846

847

848

849

850

851

852

853

854

855

856

857

858

859

860

861

862

863

864

865

866

867

868

869

870

871

872

873

874

875

876

877

Systems 38:1543-1556. DOI: 10.1109/TCAD.2018.2852752.

Li B, Najafi MH, Yuan B, Lilja DJ. 2018b. Quantized Neural Networks with New Stochastic Multipliers. Proceedings - International Symposium on Quality Electronic Design, ISQED 2018-March:376-382. DOI: 10.1109/ISQED.2018.8357316.

Li B, Qin Y, Yuan B, Lilja DJ. 2017a. Neural Network Classifiers using Stochastic Computing with a Hardware-Oriented Approximate Activation Function. Proceedings - 35th IEEE International Conference on Computer Design, ICCD 2017:97-104. DOI: 10.1109/ICCD.2017.23.

Li J, Yuan Z, Li Z, Ding C, Ren A, Qiu Q, Draper J, Wang Y. 2017b. Hardware-Driven Nonlinear Activation for Stochastic Computing Based Deep Convolutional Neural Networks. Proceedings of the International Joint Conference on Neural Networks 2017May:1230-1236. DOI: 10.1109/IJCNN.2017.7965993.

Li J, Yuan Z, Li Z, Ren A, Ding C, Draper J, Nazarian S, Qiu Q, Yuan B, Wang Y. 2017c. Normalization and dropout for stochastic computing-based deep convolutional neural networks. Integration 65:395-403. DOI: 10.1016/j.vlsi.2017.11.002.

Liew SS, Khalil-Hani M, Ahmad Radzi S, Bakhteri R. 2016. Gender Classification: A Convolutional Neural Network Approach. Turkish Journal of Electrical Engineering and Computer Sciences 24:1248-1264. DOI: 10.3906/elk-1311-58.

Liu Z, Dou Y, Jiang J, Xu J. 2017. Automatic Code Generation of Convolutional Neural Networks in FPGA Implementation. Proceedings of the 2016 International Conference on Field-Programmable Technology, FPT 2016:61-68. DOI: 10.1109/FPT.2016.7929190.

Ma X, Zhang Y, Yuan G, Ren A, Li Z, Han J, Hu J, Wang Y. 2018. An Area and Energy Efficient Design of Domain-Wall Memory-Based Deep Convolutional Neural Networks using Stochastic Computing. Proceedings - International Symposium on Quality Electronic Design, ISQED 2018-March:314-321. DOI: 10.1109/ISQED.2018.8357306.

Mittal S. 2020. A survey of FPGA-based accelerators for convolutional neural networks. Neural Computing and Applications 32:1109-1139. DOI: 10.1007/s00521-018-3761-1.

Najafi MH, Li P, Lilja DJ, Qian W, Bazargan K, Riedel M. 2017. A Reconfigurable Architecture with Sequential Logic-Based Stochastic Computing. ACM Journal on Emerging Technologies in Computing Systems 13. DOI: 10.1145/3060537.

Naveen S, Kounte MR. 2019. Key Technologies and challenges in IoT Edge Computing. 2019 Third International conference on I-SMAC (IoT in Social, Mobile, Analytics and Cloud) (ISMAC):61-65. DOI: 10.1109/I-SMAC47947.2019.9032541.

Noronha DH, Salehpour B, Wilton SJE. 2019. Leflow: Enabling Flexible FPGA High-Level Synthesis of Tensorflow Deep Neural Networks. 5th International Workshop on FPGAs for Software Programmers, FSP 2018, co-located with International Conference on Field Programmable Logic and Applications, FPL 2018:46-53.

Nurvitadhi E, Sheffield D, Sim J, Mishra A, Venkatesh G, Marr D. 2017. Accelerating Binarized Neural Networks: Comparison of FPGA, CPU, GPU, and ASIC. Proceedings of the 2016 International Conference on Field-Programmable Technology, FPT 2016:77-84. DOI: 10.1109/FPT.2016.7929192.

Ren A, Li Z, Ding C, Qiu Q, Wang Y, Li J, Qian X, Yuan B. 2017. SC-DCNN: Highly-Scalable Deep Convolutional Neural Network Using Stochastic Computing. International Conference on Architectural Support for Programming Languages and Operating Systems ASPLOS Part F1271:405-418. DOI: 10.1145/3037697.3037746.

Ren A, Li Z, Wang Y, Qiu Q, Yuan B. 2016. Designing Reconfigurable Large-Scale Deep

Peer] Comput. Sci. reviewing PDF | (CS-2020:07:51446:1:1:NEW 23 Sep 2020) 
878

879

880

881

882

883

884

885

886

887

888

889

890

891

892

893

894

895

896

897

898

899

900

901

902

903

904

905

906

907

908

909

910

911

912
Learning Systems Using Stochastic Computing. 2016 IEEE International Conference on Rebooting Computing, ICRC 2016 - Conference Proceedings. DOI:

10.1109/ICRC.2016.7738685.

Salehi SA. 2020. Low-Cost Stochastic Number Generators for Stochastic Computing. IEEE Transactions on Very Large Scale Integration (VLSI) Systems 28:992-1001. DOI: 10.1109/TVLSI.2019.2963678.

Sim H, Lee J. 2017. A New Stochastic Computing Multiplier with Application to Deep Convolutional Neural Networks. Proceedings - Design Automation Conference Part 12828:1-6. DOI: 10.1145/3061639.3062290.

Sim H, Nguyen D, Lee J, Choi K. 2017. Scalable Stochastic-Computing Accelerator for Convolutional Neural Networks. Proceedings of the Asia and South Pacific Design Automation Conference, ASP-DAC:696-701. DOI: 10.1109/ASPDAC.2017.7858405.

Simons T, Lee DJ. 2019. A Review of Binarized Neural Networks. Electronics (Switzerland) 8. DOI: 10.3390/electronics8060661.

Xie Y, Liao S, Yuan B, Wang Y, Wang Z. 2017. Fully-Parallel Area-Efficient Deep Neural Network Design Using Stochastic Computing. IEEE Transactions on Circuits and Systems II: Express Briefs 64:1382-1386. DOI: 10.1109/TCSII.2017.2746749.

Yang M, Li B, Lilja DJ, Yuan B, Qian W. 2018. Towards Theoretical Cost Limit of Stochastic Number Generators for Stochastic Computing. Proceedings of IEEE Computer Society Annual Symposium on VLSI, ISVLSI 2018-July:154-159. DOI: 10.1109/ISVLSI.2018.00037.

Yu J, Kim K, Lee J, Choi K. 2017. Accurate and Efficient Stochastic Computing Hardware for Convolutional Neural Networks. Proceedings - 35th IEEE International Conference on Computer Design, ICCD 2017:105-112. DOI: 10.1109/ICCD.2017.24.

Zhakatayev A, Lee S, Sim H, Lee J. 2018. Sign-Magnitude SC: Getting 10X Accuracy for Free in Stochastic Computing for Deep Neural Networks. Proceedings - Design Automation Conference Part F1377:1-6. DOI: 10.1145/3195970.3196113.

Zhang Y, Zhang X, Song J, Wang Y, Huang R, Wang R. 2019. Parallel Convolutional Neural Network (CNN) Accelerators Based on Stochastic Computing. In: IEEE Workshop on Signal Processing Systems, SiPS: Design and Implementation. 19-24. DOI: 10.1109/SiPS47522.2019.9020615.

Zhu S, Dong X, Su H. 2019. Binary Ensemble Neural Network: More Bits per Network or More Networks per Bit? Proceedings of the IEEE Computer Society Conference on Computer Vision and Pattern Recognition 2019-June:4918-4927. DOI: 10.1109/CVPR.2019.00506. 
Figure 1

Process of SC and its elements.

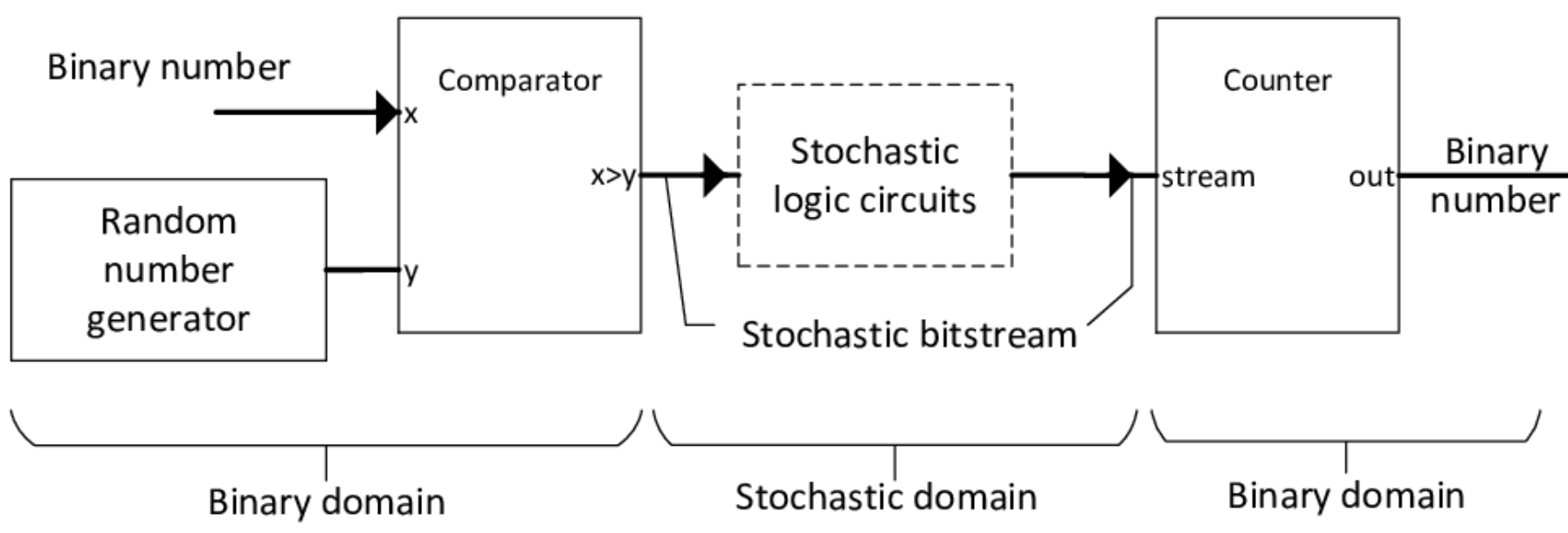


Figure 2

SC arithmetic operation.

(A)AND gate as SC unipolar multiplier. (B) MUX as SC scaled adder. (C) Uncorrelated bit streams give accurate output. (D) Correlated bit streams give inaccurate output.

A

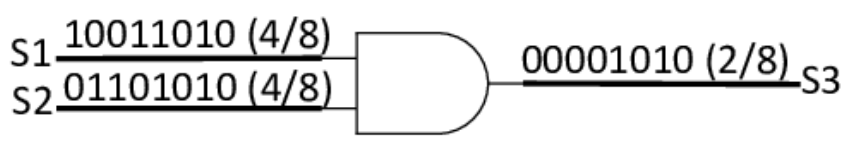

S1 $\frac{10011010(4 / 8)}{01101010(4 / 8)} \longrightarrow \underline{00001010(2 / 8)}$ S3

C

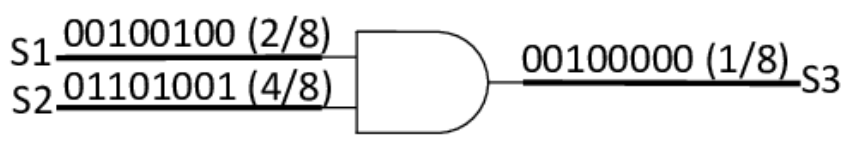

B

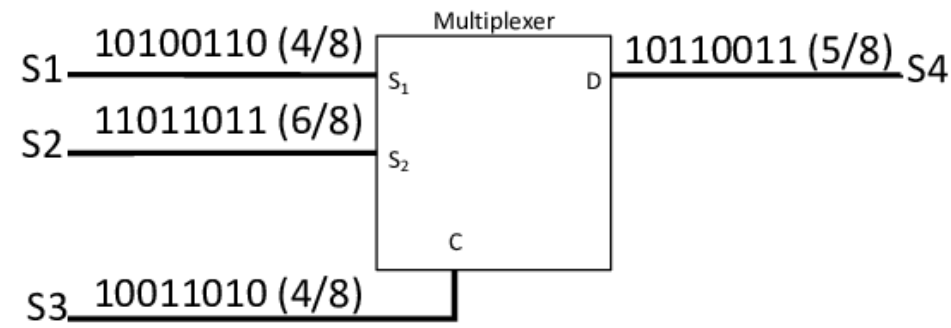

D

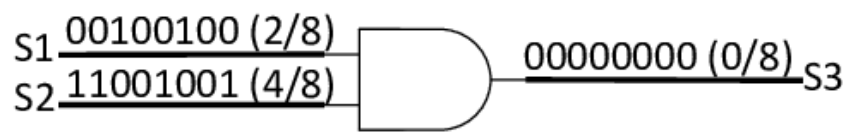


Figure 3

SNG components.

(A) RNG with LFSR. (B) True comparator. (C) WBG. 


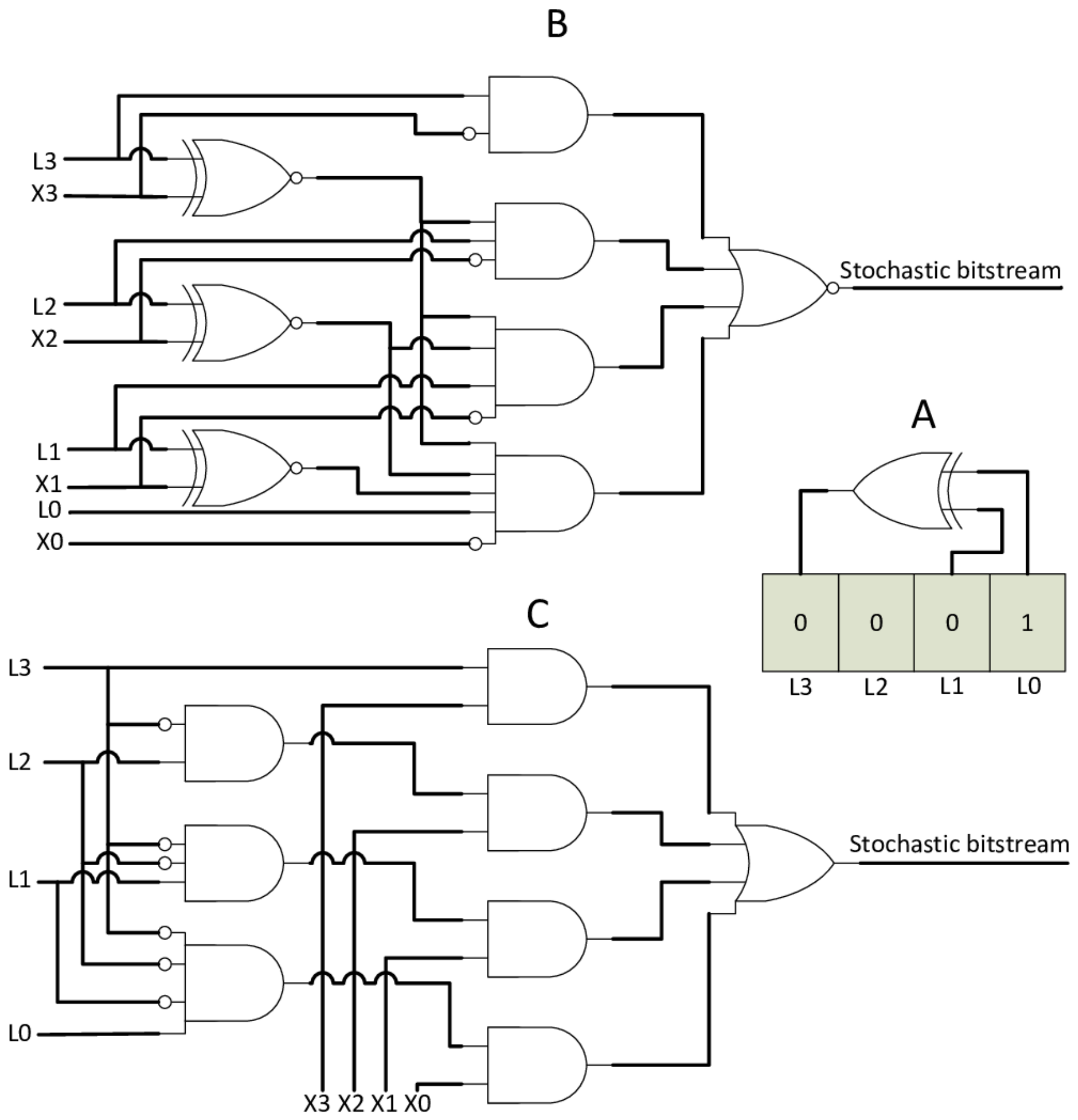


Figure 4

SC with advance arithmetic operations.

(A) Stochastic squaring with D flip-flop. (B) K-state FSM for Stanh function which will be widely utilised in SC CNN.
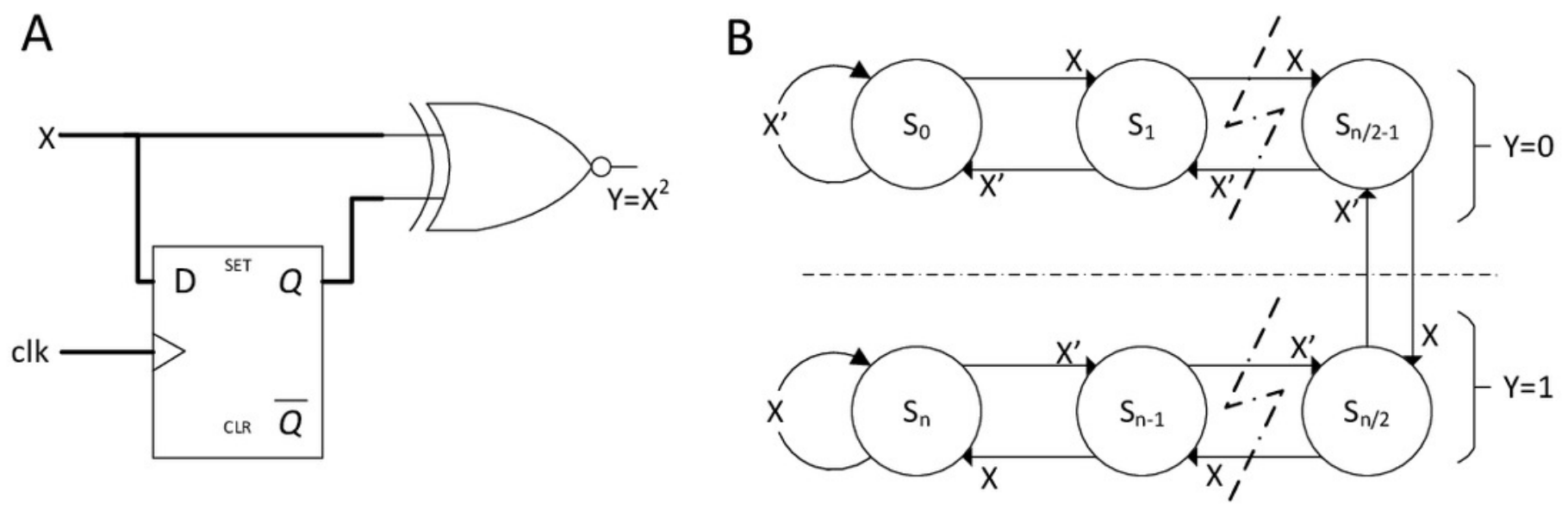
Figure 5

SC divider circuits.

(A) Former gradient descent unipolar divider. (B) Former SC bipolar divider. (C) Newer SC unipolar divider by exploiting correlation. (D) Newer SC bipolar divider by adding sign information.
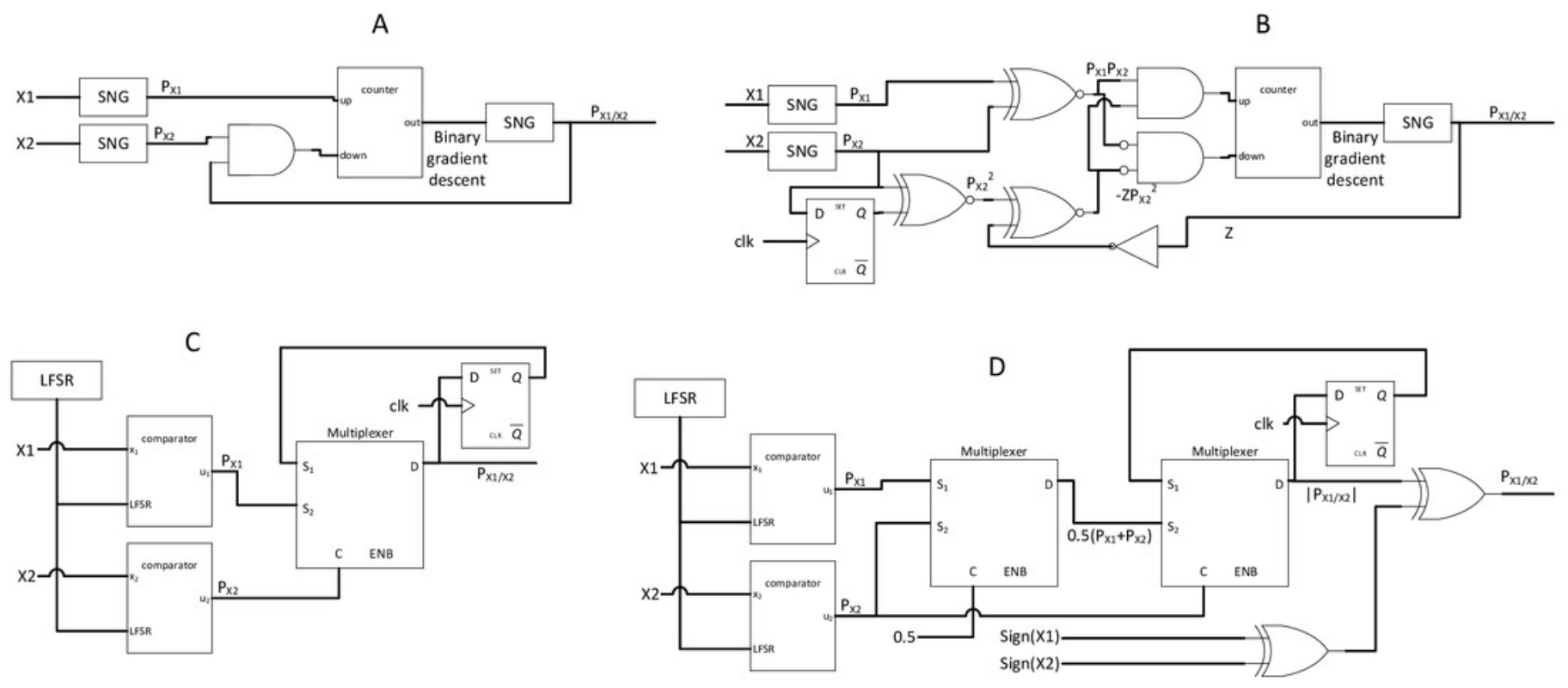
Figure 6

CNN's convolution and activation

(A) Matrix convolution. (B) Neural network model after the convolution. (C) Architecture of classical LeNet-5 CNN.
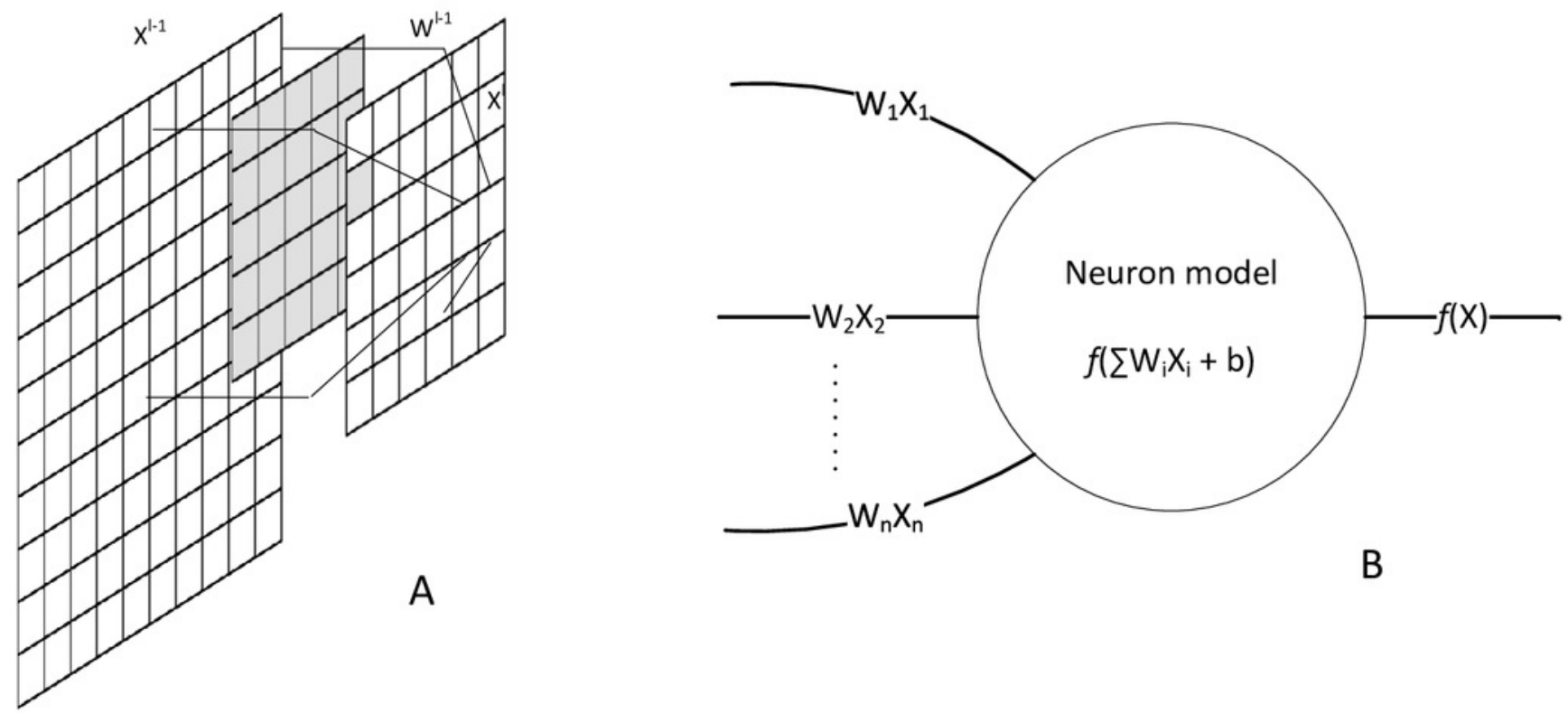

B

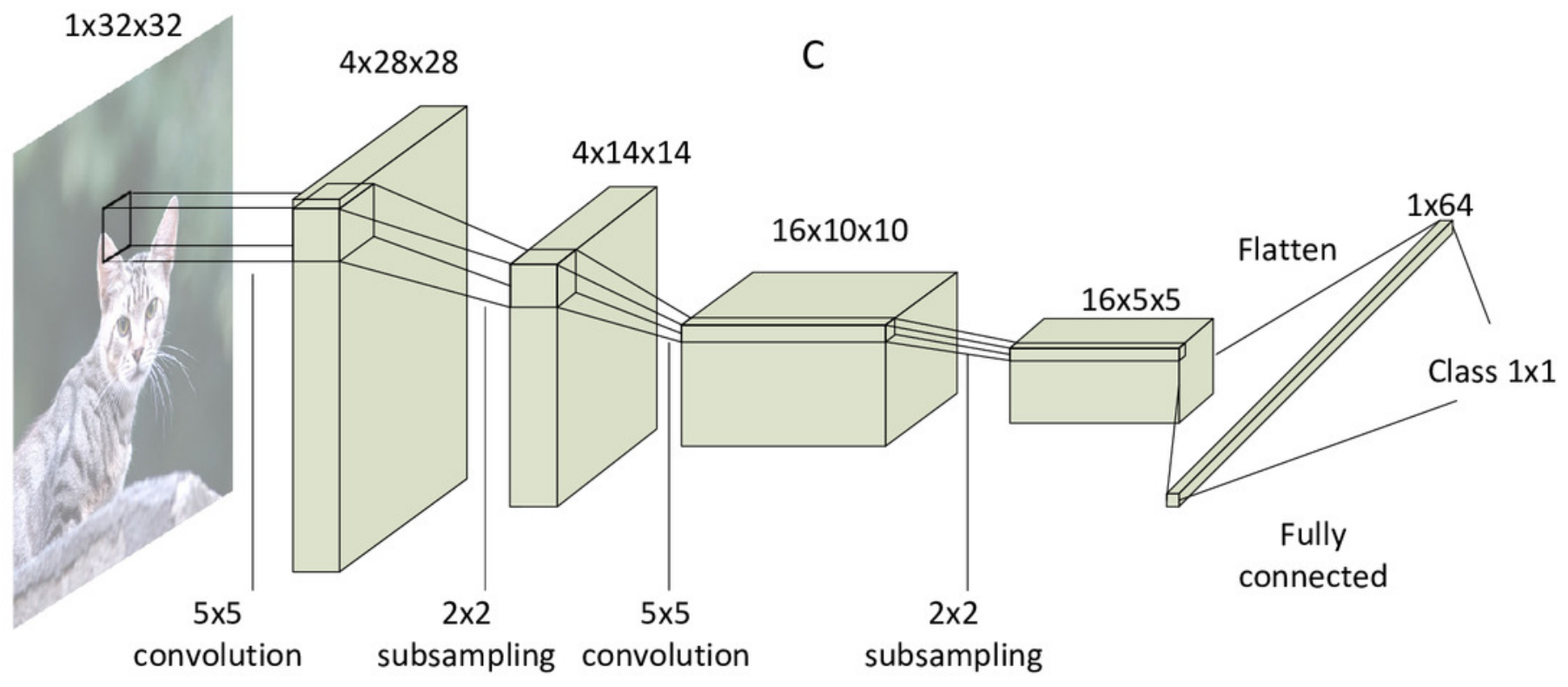


Figure 7

Integral SC methodology.

(A) High precision stochastic number can be represented with shorter stream length with integer value. (B) Binary radix multiplier as integral SC scaled adder. (C) Modified MUX as integral SC multiplier. (D) Integer SC neuron block.
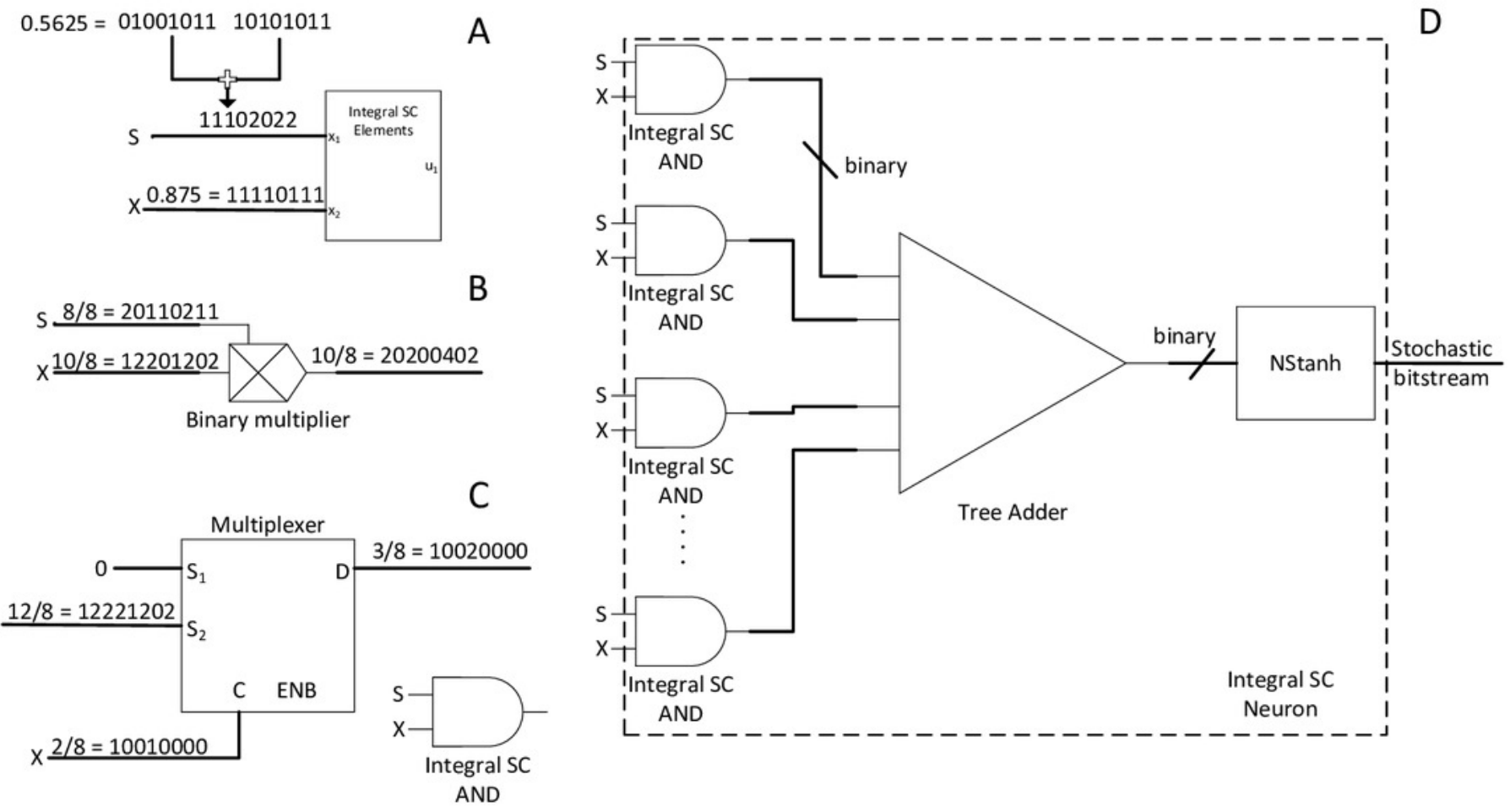
Figure 8

ESL arithmetic unit.

(A) ESL multiplier. (B) ESL divider by crossing multiplication. (B) ESL adder and subtractor circuit.

A
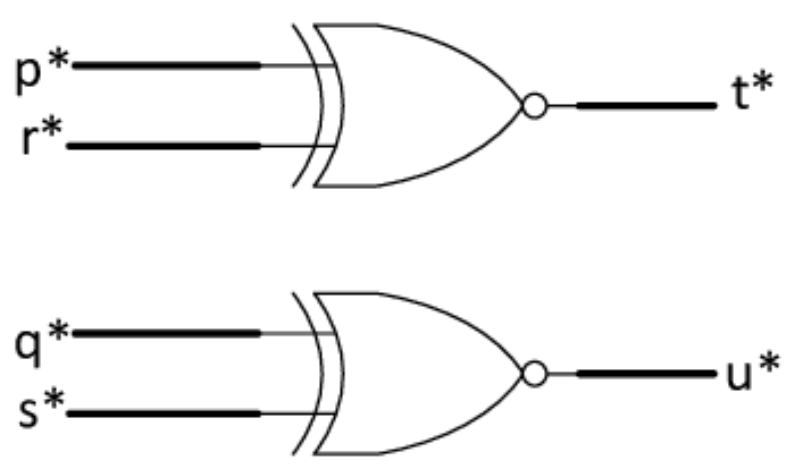

ESL multiplier
B
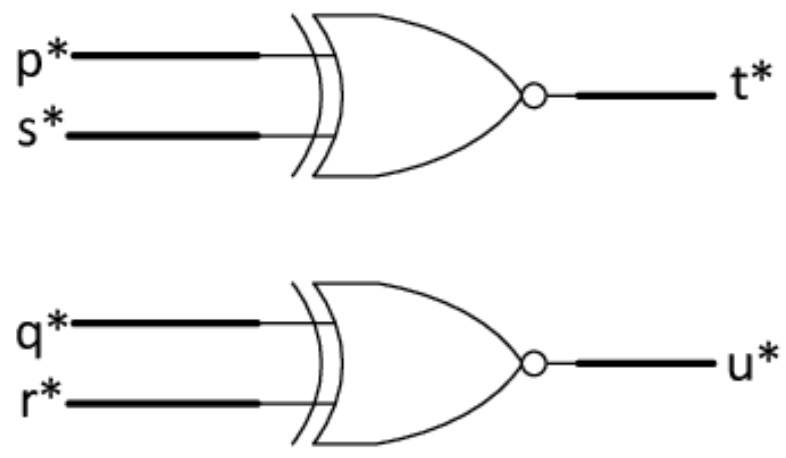

ESL divider

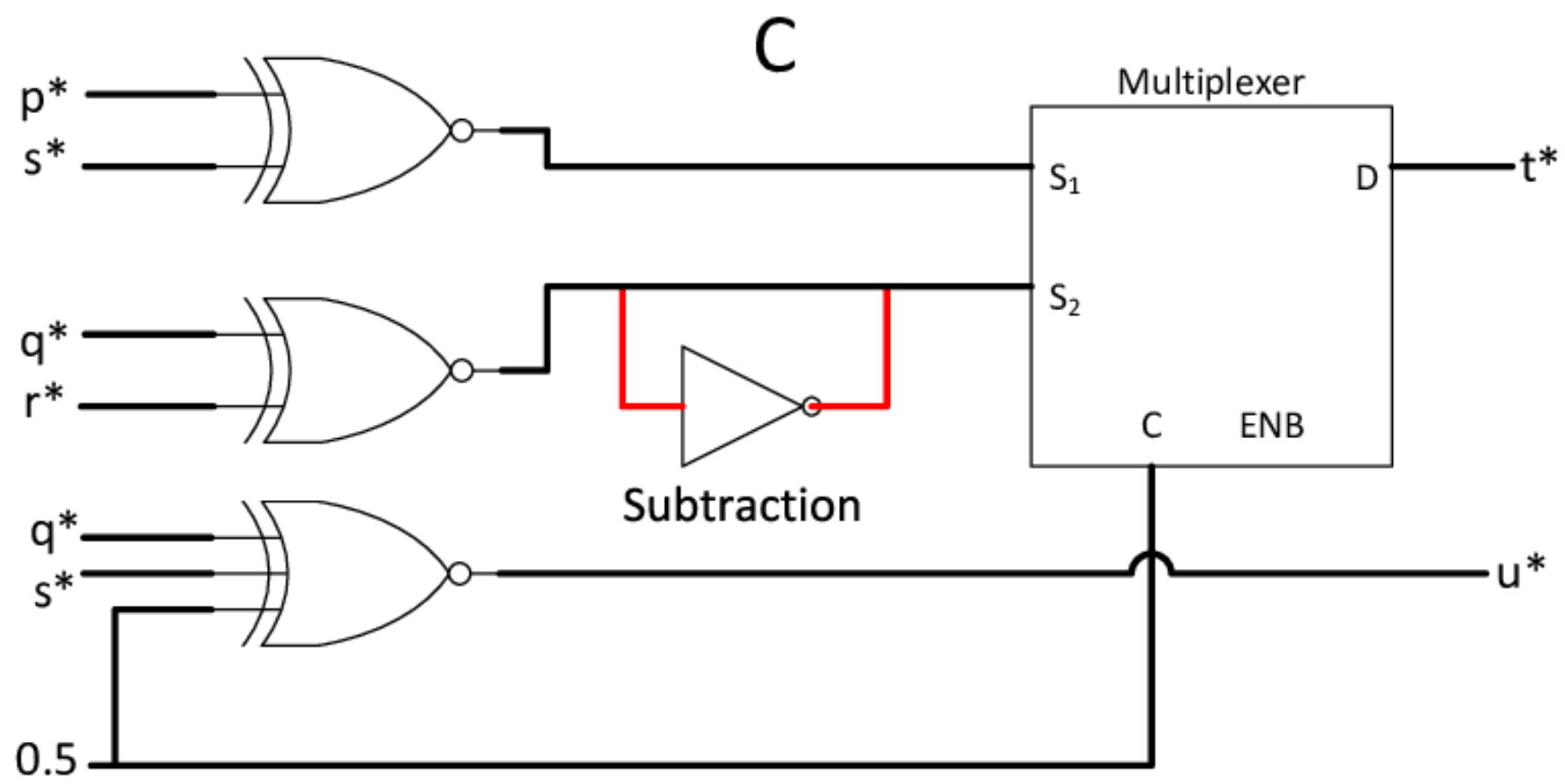

ESL Adder/Subtractor 
Figure 9

SC bitstream accumulation.

(A) APC. (B) Accurate parallel counter. (C) Accumulation and Btanh activation workflow.
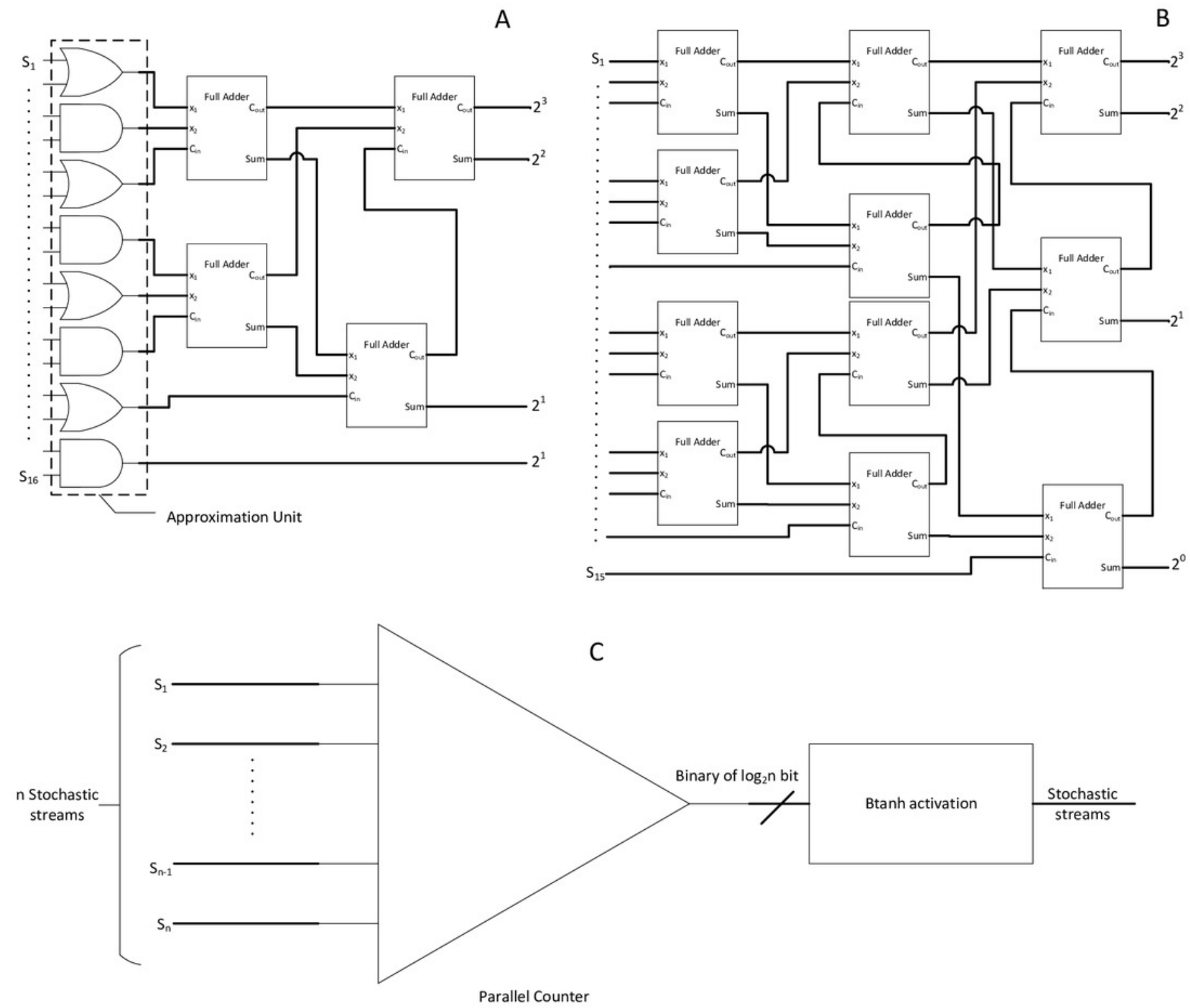
Figure 10

SC pooling function.

(A) $2 \times 2$ average pooling with cascaded MUX adder. (B) hardware-oriented approximate max pooling circuit. (C) Stochastic MAX function, cascading them will create pure SC max pool block.
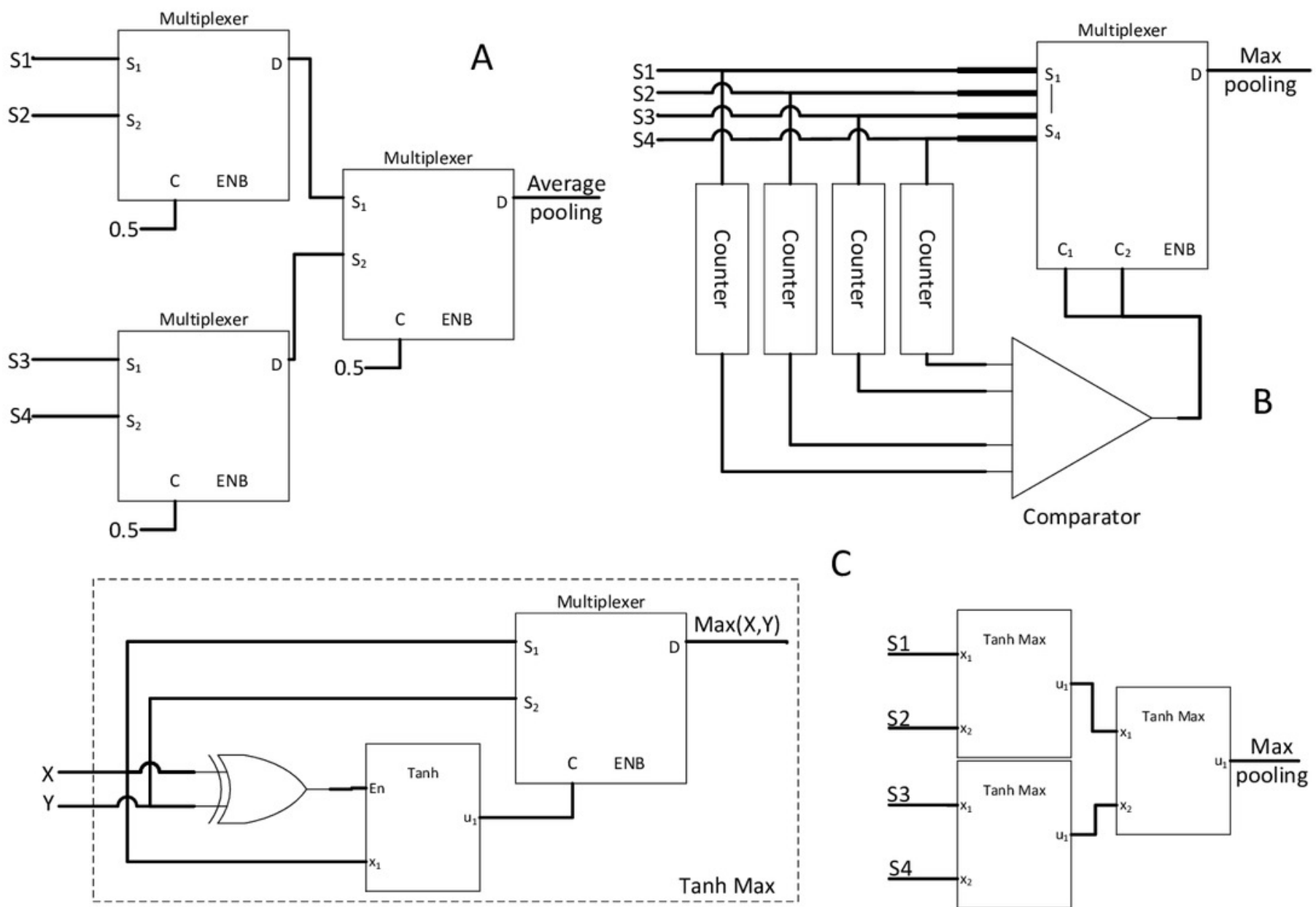

C

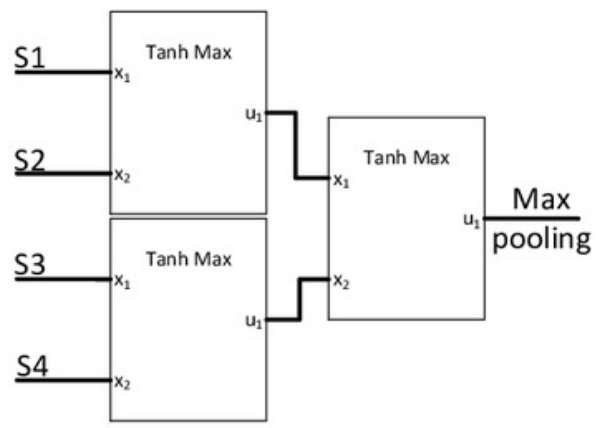


Figure 11

Other SC activation functions.

(A) ReLU activation function. (B) SC sigmoid activation function with bias input.
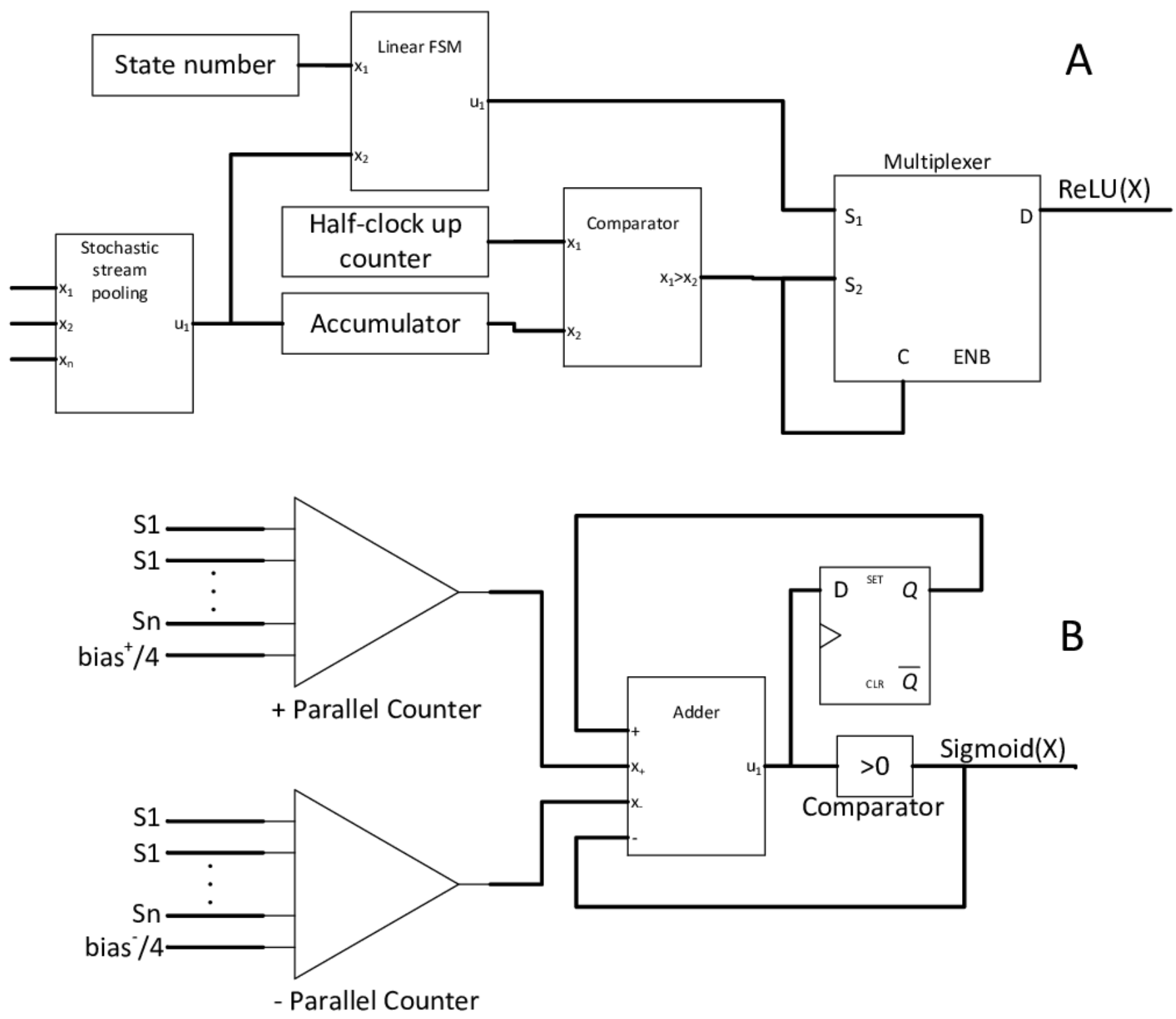
Figure 12

\section{Normalisation unit in SC CNN.}

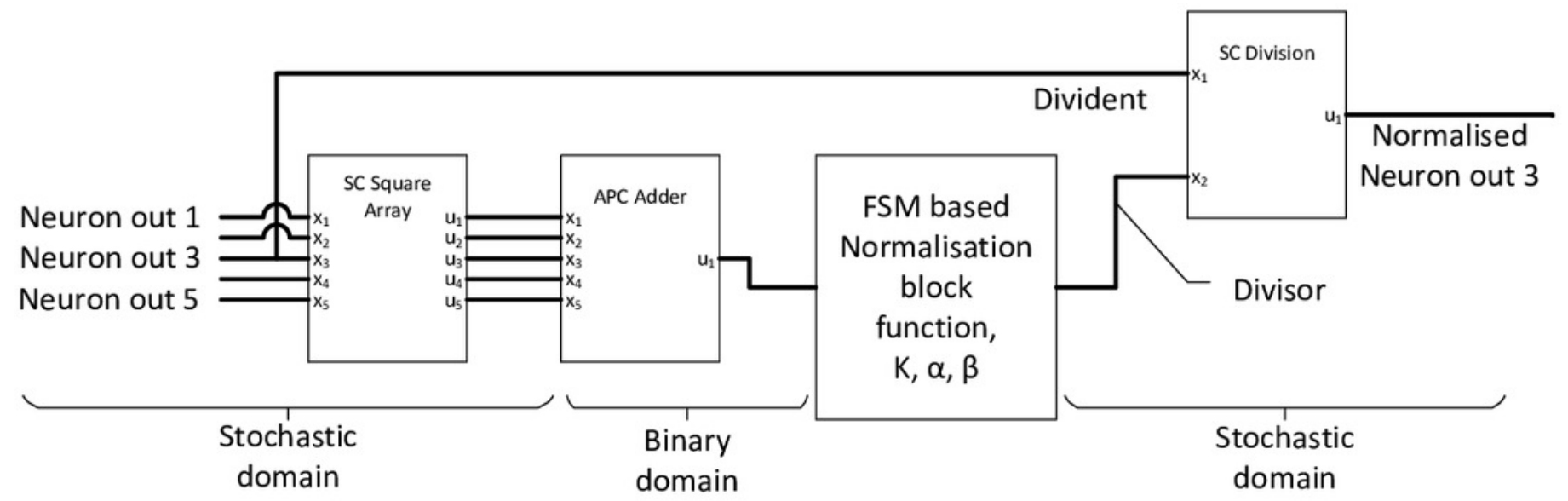




\section{Figure 13}

Binary interlaced SC, where SC is used as MAC accelerator.

(A) SC MAC unit block. (B) SC MAC optimisation by cutting off SC early with advancing weight bits. (C) Novel SNG with MUX and FSM. 


\section{A}

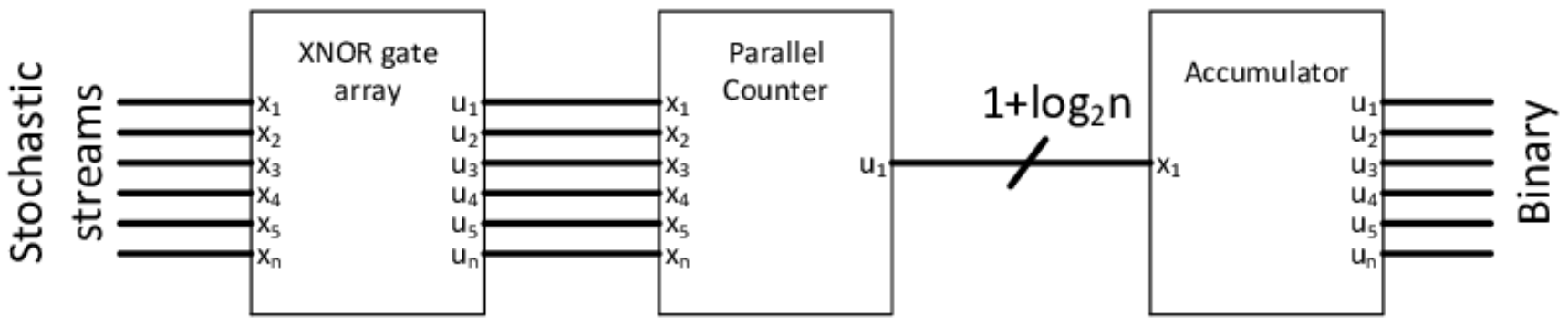

B
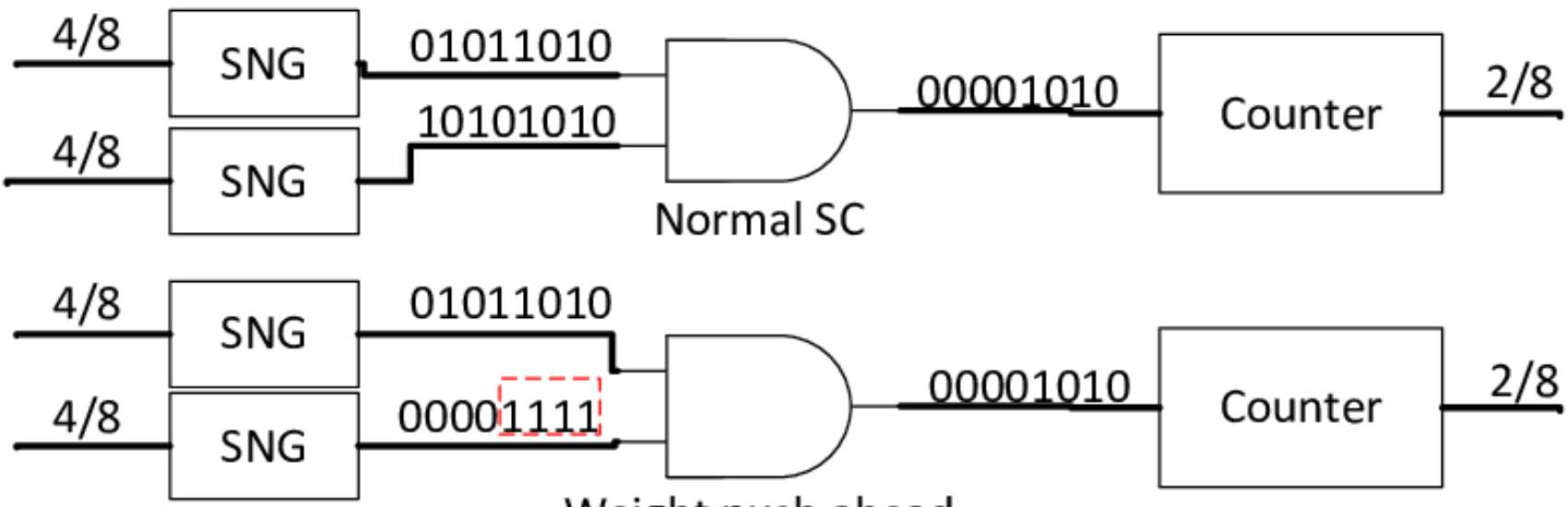

Weight push ahead
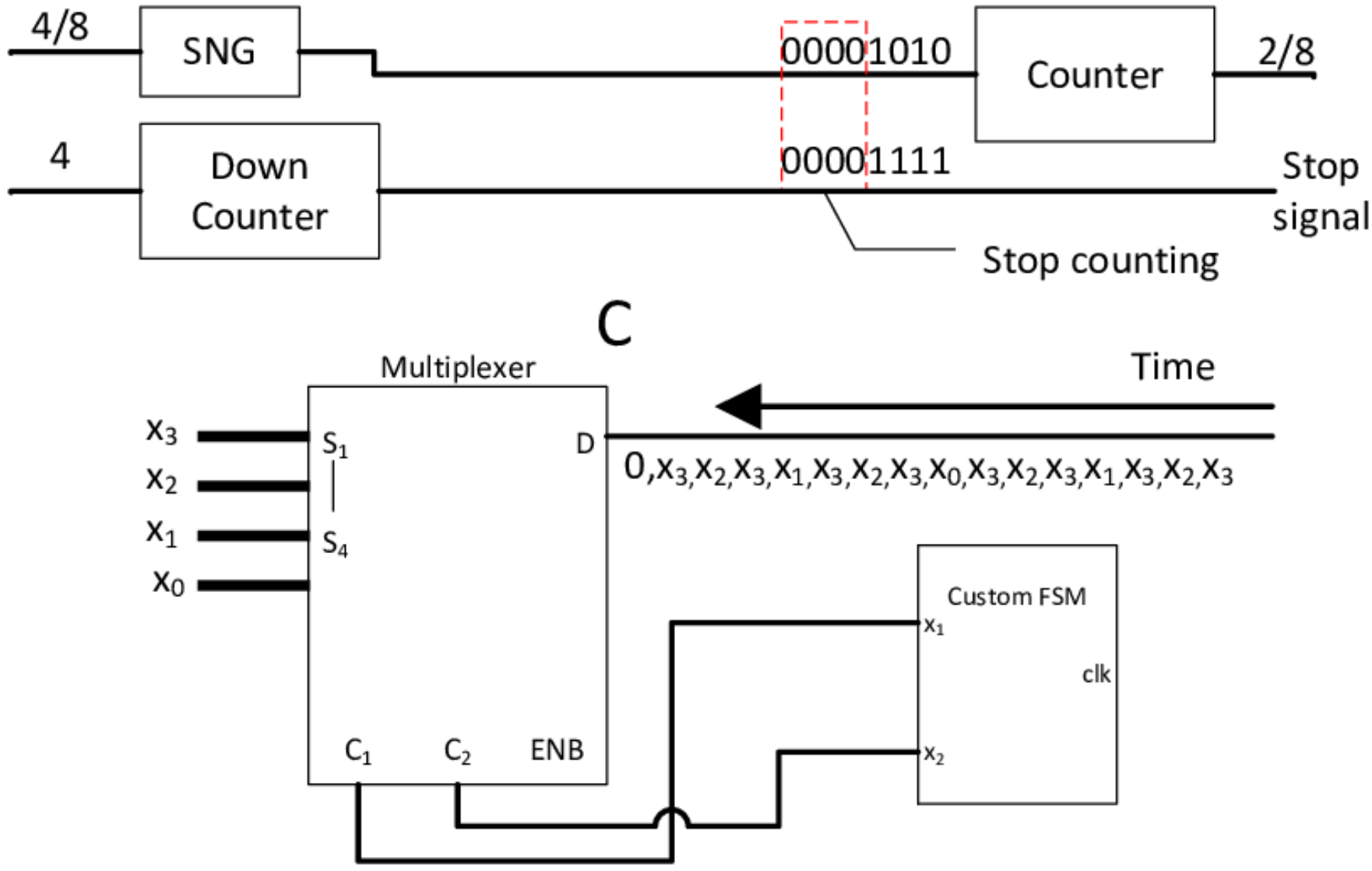


\section{Figure 14}

Differential MAC. Major overhauling to the SN MAC with counter and differential weight control indexing to pipeline the SC MAC computation. 


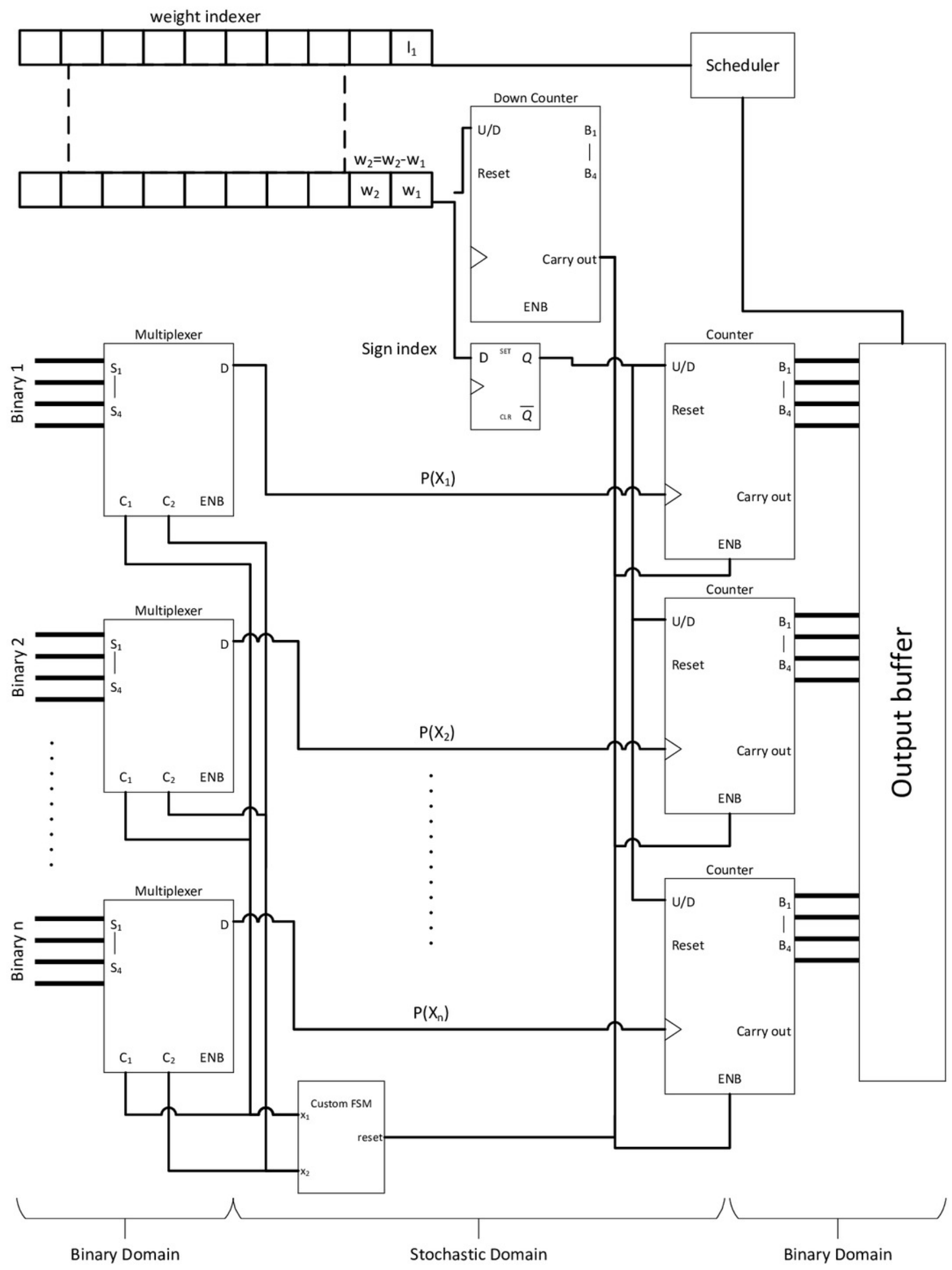




\section{Figure 15}

Stochastic quantisation accumulation with SUC adder.

(A) The different information portion of the stochastic stream could be encoded into a single stream by OR-ing the required bitstream asynchronously. (B) SUC paired with SC sigmoid activation function. 

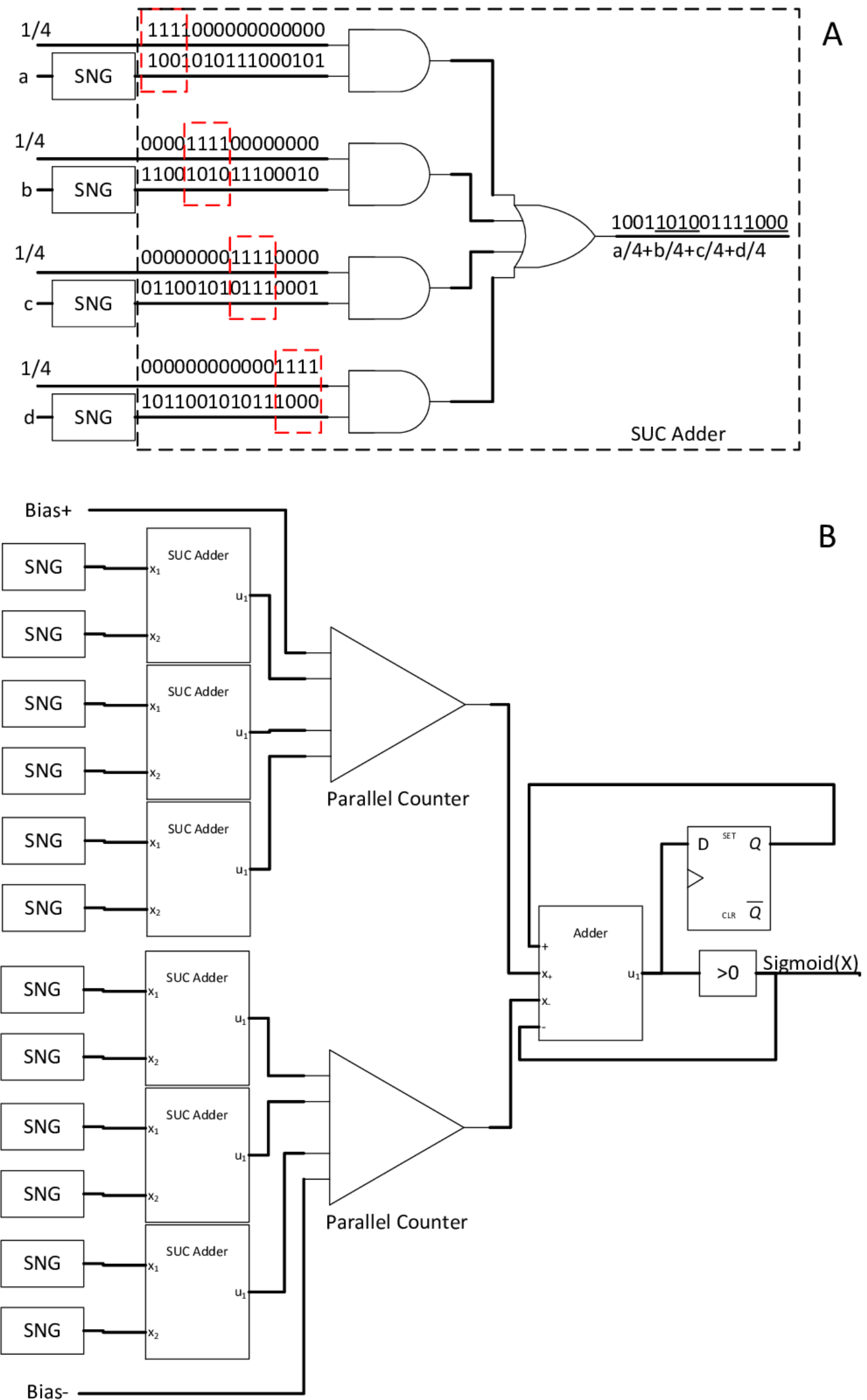
Figure 16

ASC with thermometer coding.

(A) Implementation of ASC on thermometer-encoded SC circuit, eliminating the need for ADC and memory components. (B) Thermometer coding could be utilised for SNGs.

A

\begin{tabular}{|c|c|c|c|}
\hline $\mathrm{Vin}$ & $\mathrm{Y}_{2}$ & $\mathrm{Y}_{1}$ & $\mathrm{Y}_{0}$ \\
\hline$<\mathrm{V} / 4$ & 0 & 0 & 0 \\
\hline$<\mathrm{V} / 2$ & 0 & 0 & 1 \\
\hline$<3 \mathrm{~V} / 4$ & 0 & 1 & 1 \\
\hline$>3 \mathrm{~V} / 4$ & 1 & 1 & 1 \\
\hline
\end{tabular}

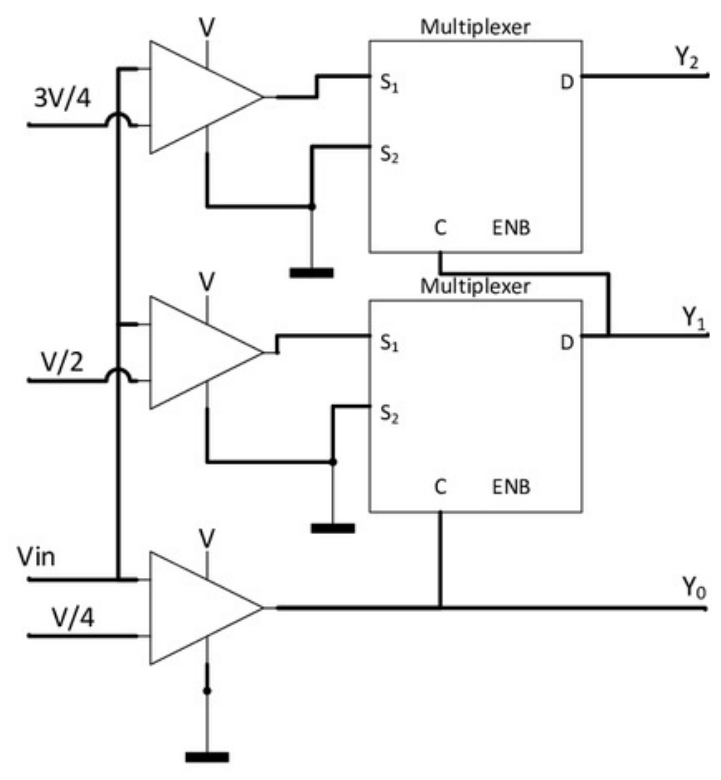

B

\begin{tabular}{|c|c|c|c|c|c|c|c|c|c|}
\hline \multicolumn{4}{|c|}{ Binary } & \multicolumn{7}{|c|}{ Thermometer code } \\
\hline $\mathbf{X}_{\mathbf{2}}$ & $\mathrm{X}_{1}$ & $\mathrm{X}_{\mathbf{0}}$ & $\mathrm{Y}_{6}$ & $\mathrm{Y}_{5}$ & $\mathrm{Y}_{4}$ & $\mathrm{Y}_{\mathbf{3}}$ & $\mathrm{Y}_{\mathbf{2}}$ & $\mathrm{Y}_{\mathbf{1}}$ & $\mathrm{Y}_{\mathbf{0}}$ \\
\hline 0 & 0 & 0 & 0 & 0 & 0 & 0 & 0 & 0 & 0 \\
\hline 0 & 0 & 1 & 0 & 0 & 0 & 0 & 0 & 0 & 1 \\
\hline 0 & 1 & 0 & 0 & 0 & 0 & 0 & 0 & 1 & 1 \\
\hline 0 & 1 & 1 & 0 & 0 & 0 & 0 & 1 & 1 & 1 \\
\hline 1 & 0 & 0 & 0 & 0 & 0 & 1 & 1 & 1 & 1 \\
\hline 1 & 0 & 1 & 0 & 0 & 1 & 1 & 1 & 1 & 1 \\
\hline 1 & 1 & 0 & 0 & 1 & 1 & 1 & 1 & 1 & 1 \\
\hline 1 & 1 & 1 & 1 & 1 & 1 & 1 & 1 & 1 & 1 \\
\hline
\end{tabular}

$x_{2}-$

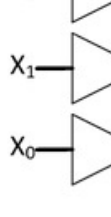




\section{Figure 17}

SC BNN methodology.

(A) The similarity of SC and BNN in terms of logic gate utilisation. (B) Usual configuration in binary BNN. (C) SC BNN first layer binary image conversion in SC BNN.
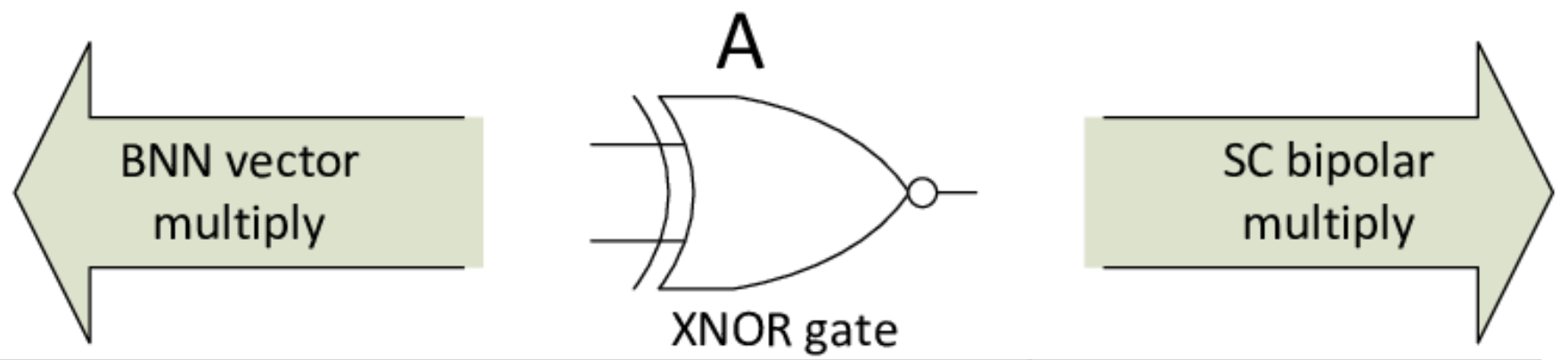

\begin{tabular}{|c|c|c|}
\hline \multicolumn{2}{|c|}{ Encoding (value) } & XNOR multiply \\
\hline $0(-1)$ & $0(-1)$ & $1(+1)$ \\
\hline $0(-1)$ & $1(+1)$ & $0(-1)$ \\
\hline $1(+1)$ & $0(-1)$ & $0(-1)$ \\
\hline $1(+1)$ & $1(+1)$ & $1(+1)$ \\
\hline
\end{tabular}

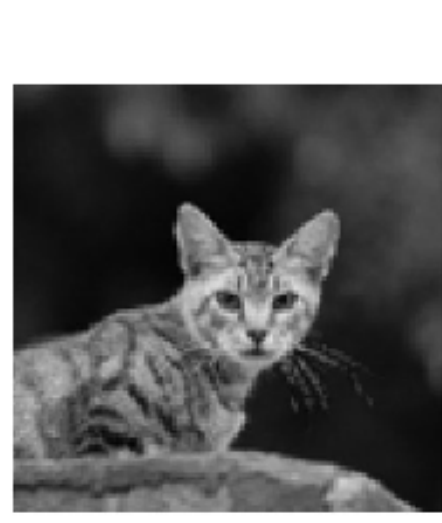

B

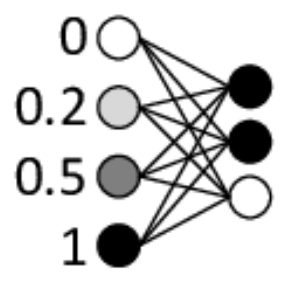

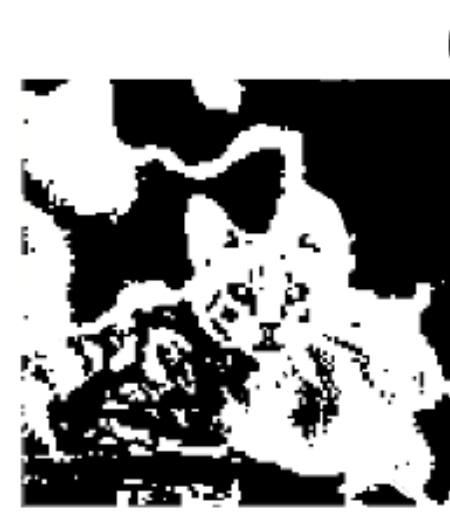

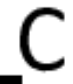

...0100101 ...1011010 1..1101100○ 
Figure 18

Process flow in SC CNN and the internal computing domain interchange.

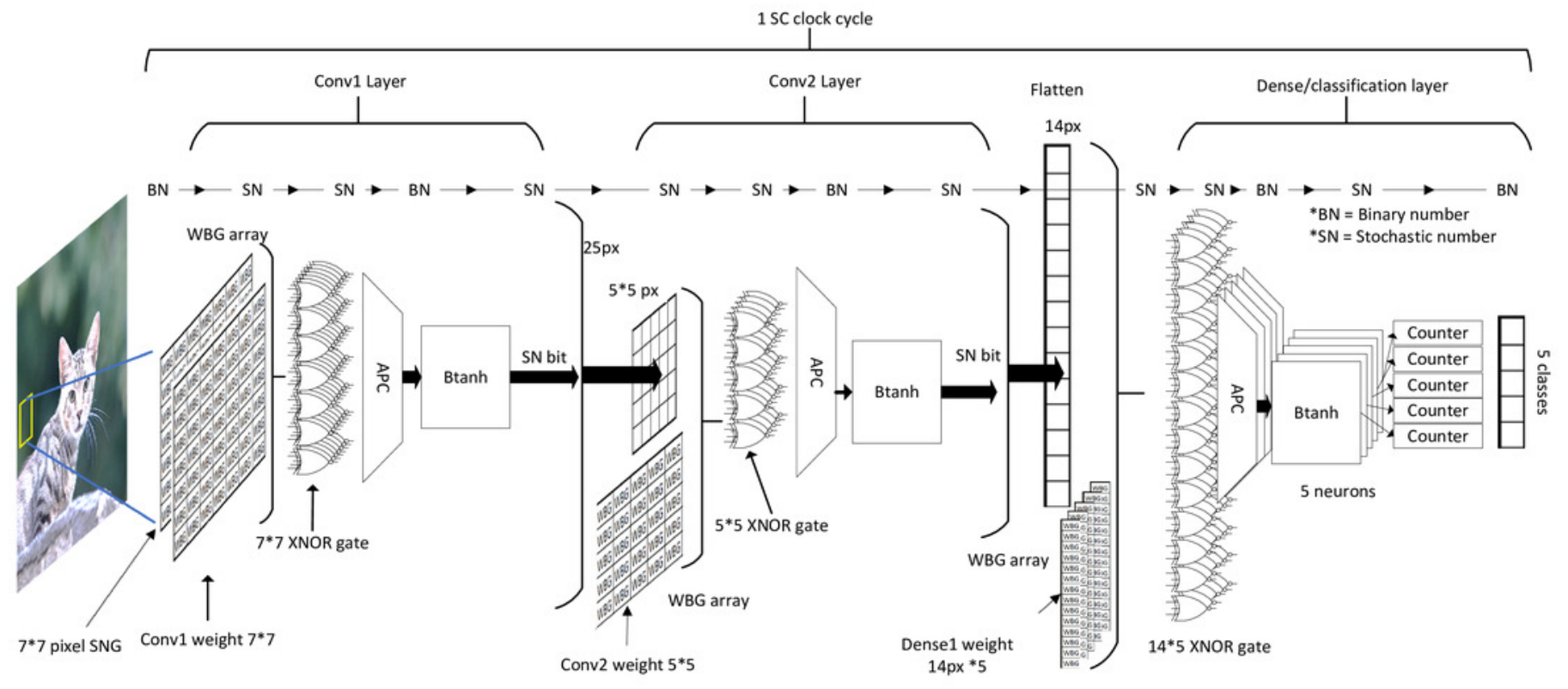


Figure 19

Process flow in SC BNN, stochastic image generation methodology and the internal computing domain interchange.

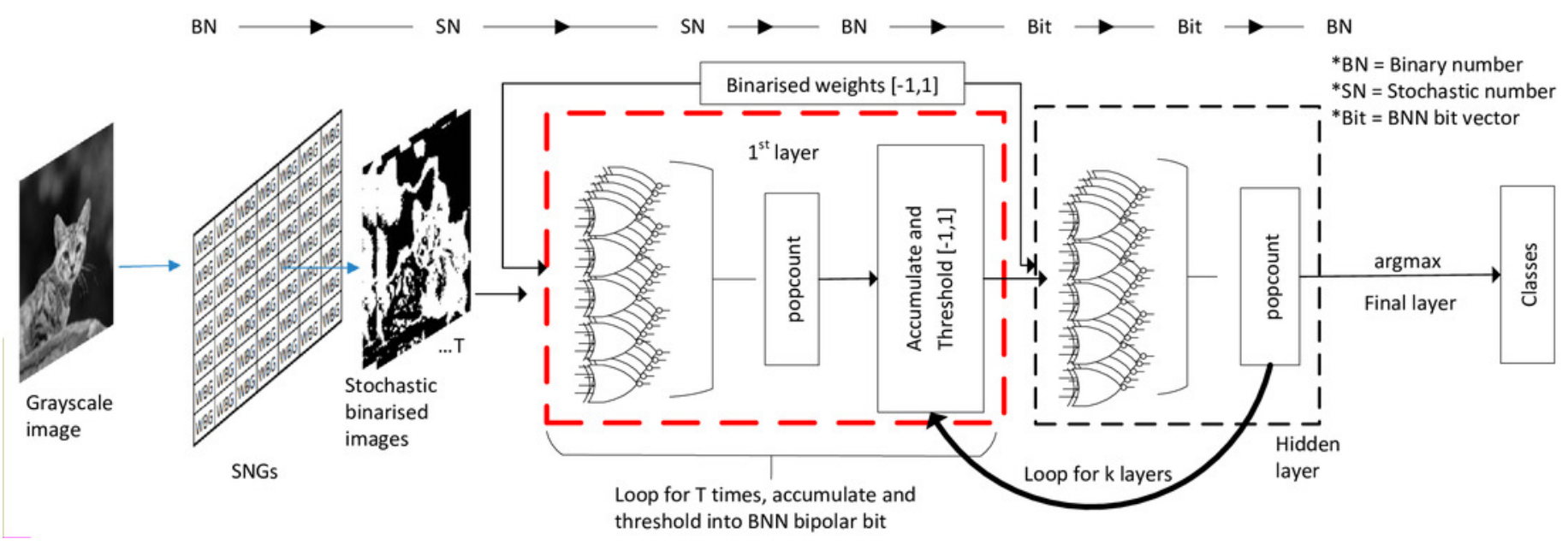




\section{Table $\mathbf{1}$ (on next page)}

Performance difference across SC and conventional binary domain. 


\begin{tabular}{|c|c|c|c|c|c|c|c|}
\hline CNN Model & Platform & Year & Method & $\begin{array}{l}\text { Area }( \\
\left.\mathrm{mm}^{2}\right)\end{array}$ & $\begin{array}{c}\text { Power }(\mathrm{W}) \\
\text { or energy } \\
(\mathrm{nJ})\end{array}$ & $\begin{array}{c}\text { Accuracy } \\
(\%)\end{array}$ & $\begin{array}{c}\text { Energy } \\
\text { efficiency } \\
\text { (images/J) } \\
\text { or } \\
\text { (GOPS/W) }\end{array}$ \\
\hline \multirow{5}{*}{ Lenet-5 } & $\mathrm{CPU}$ & 2009 & Software & 263 & $156 \mathrm{~W}$ & 99.17 & 4.2 \\
\hline & GPU & 2011 & Software & 520 & $202.5 \mathrm{~W}$ & 99.17 & 3.2 \\
\hline & ASIC & 2016 & $\begin{array}{c}\text { SC } 256 \\
\text { bit (Ren } \\
\text { et al., } \\
2017) \\
\end{array}$ & 36.4 & $3.53 \mathrm{~W}$ & 98.26 & 221,287 \\
\hline & ASIC & 2018 & $\begin{array}{l}\text { SC 128bit } \\
\text { (Li et al., } \\
\text { 2018a) } \\
\end{array}$ & 22.9 & $2.6 \mathrm{~W}$ & 99.07 & $1,231,971$ \\
\hline & ASIC & 2018 & $\begin{array}{c}\text { SC DWM } \\
\text { 128bit } \\
\text { (Ma et al., } \\
2018) \\
\end{array}$ & 19.8 & $0.028 \mathrm{~W}$ & 98.94 & - \\
\hline \multirow{3}{*}{$\begin{array}{c}\text { AlexNet } \\
\text { (last second } \\
\text { layer) }\end{array}$} & $\mathrm{CPU}$ & 2009 & Software & 263 & $156 \mathrm{~W}$ & - & 0.9 \\
\hline & GPU & 2011 & Software & 520 & $202.5 \mathrm{~W}$ & - & 2.8 \\
\hline & ASIC & 2018 & $\begin{array}{l}\text { SC 128bit } \\
\text { (Li et al., } \\
\text { 2018a) }\end{array}$ & 24.7 & $1.9 \mathrm{~W}$ & - & $1,326,400$ \\
\hline \multirow{3}{*}{$\begin{array}{l}\text { Custom } \\
\text { (3x3filter) }\end{array}$} & ASIC & 2015 & Binary & 5.429 & $3.287 \mathrm{~mW}$ & - & - \\
\hline & ASIC & 2017 & $\mathrm{SC} \mathrm{MAC}$ & 1.408 & $1.369 \mathrm{~mW}$ & - & - \\
\hline & ASIC & 2019 & $\begin{array}{c}\mathrm{SC} \\
\mathrm{DMAC}\end{array}$ & 1.439 & $1.393 \mathrm{~mW}$ & - & - \\
\hline \multirow{2}{*}{$\begin{array}{c}\text { Custom } \\
\text { (Ardakani } \\
\text { et al., 2017) }\end{array}$} & $\mathrm{ASIC}$ & 2017 & Binary & - & $380 \mathrm{~nJ}$ & 97.7 & - \\
\hline & ASIC & 2017 & $\begin{array}{c}\text { Integral } \\
\text { SC }\end{array}$ & - & $299 \mathrm{~nJ}$ & 97.73 & - \\
\hline \multirow{2}{*}{$\begin{array}{l}\text { ConvNet } \\
\text { for MNIST }\end{array}$} & ASIC & 2015 & Binary & 0.98 & $0.236 \mathrm{~W}$ & - & $\begin{array}{c}1158.11 \\
\text { GOPS/W } \\
\end{array}$ \\
\hline & ASIC & 2017 & $\mathrm{SC} \mathrm{MAC}$ & 0.43 & $0.279 \mathrm{~W}$ & - & $\begin{array}{c}5640.23 \\
\text { GOPS/W } \\
\end{array}$ \\
\hline \multirow{2}{*}{$\begin{array}{c}\text { Custom } \\
\text { (Hirtzlin et } \\
\text { al., 2019) }\end{array}$} & $\mathrm{ASIC}$ & 2019 & $\mathrm{BNN}$ & 1.95 & $220 \mathrm{~nJ}$ & 91 & - \\
\hline & ASIC & 2019 & $\mathrm{SC} B N N$ & 0.73 & $90 \mathrm{~nJ}$ & 89.6 & - \\
\hline
\end{tabular}

*GOPS = Giga operations per second 


\section{Table 2 (on next page)}

Component-wise performance comparison of SC CNN. 


\begin{tabular}{|c|c|c|c|c|c|c|}
\hline $\begin{array}{c}\mathrm{SC} \\
\mathrm{CNN} / \mathrm{BNN} \\
\text { components }\end{array}$ & Author & $\begin{array}{c}\text { Platform / } \\
\text { software }\end{array}$ & $\begin{array}{c}\text { Relative } \\
\text { accuracy } \\
(\%)\end{array}$ & $\begin{array}{l}\text { Area / } \\
\text { gate } \\
\text { count } \\
(\%)\end{array}$ & $\begin{array}{c}\text { Power / } \\
\text { Energy saving } \\
(\%)\end{array}$ & Relative to \\
\hline Integra SC & $\begin{array}{l}\text { (Ardakani } \\
\text { et al., 2017) }\end{array}$ & $\begin{array}{c}\text { FPGA \& } \\
\text { ASIC }\end{array}$ & +0.03 & -33.9 & 21.3 & $\begin{array}{l}\text { Binary } \\
\text { computing }\end{array}$ \\
\hline ESL & $\begin{array}{l}\text { (Canals et } \\
\text { al., 2016) }\end{array}$ & FPGA & -2.23 & - & - & $\begin{array}{l}\text { Binary } \\
\text { computing }\end{array}$ \\
\hline $\mathrm{APC}+\mathrm{B} \tanh$ & $\begin{array}{c}\text { (Kim, Lee } \\
\text { \& Choi, } \\
\text { 2016)(Kim } \\
\text { et al., 2016) }\end{array}$ & $\begin{array}{l}\text { Synopsys } \\
\text { Design } \\
\text { Compiler }\end{array}$ & $\begin{array}{l}-0.18 \\
-1.71 \\
\text { (EDT) }\end{array}$ & -50.0 & $\begin{array}{c}70.0 \\
76.2(\text { EDT })\end{array}$ & $\begin{array}{l}\text { Binary } \\
\text { computing }\end{array}$ \\
\hline $\begin{array}{c}\text { APC with } \\
\text { inverse adder }\end{array}$ & $\begin{array}{c}\text { (Li et al., } \\
\text { 2018a) }\end{array}$ & $\begin{array}{l}\text { Synopsys } \\
\text { Design } \\
\text { Compiler }\end{array}$ & - & -50.0 & 10.0 & $\begin{array}{l}\text { Normal } \\
\text { APC }\end{array}$ \\
\hline $\begin{array}{c}\text { SC } \\
\text { MaxPooling }\end{array}$ & $\begin{array}{l}\text { (Ren et al., } \\
\text { 2017) }\end{array}$ & $\begin{array}{l}\text { Synopsys } \\
\text { Design } \\
\text { Compiler }\end{array}$ & -0.20 & -92.7 & 98.3 & $\begin{array}{l}\text { GPU } \\
\text { computing }\end{array}$ \\
\hline $\begin{array}{l}\text { SC ReLU } \\
\text { activation }\end{array}$ & $\begin{array}{c}\text { (Li et al., } \\
\text { 2018a) }\end{array}$ & $\begin{array}{l}\text { Synopsys } \\
\text { Design } \\
\text { Compiler }\end{array}$ & -0.88 & -95.3 & 99.1 & $\begin{array}{l}\text { GPU } \\
\text { computing }\end{array}$ \\
\hline $\begin{array}{c}\mathrm{SC} \\
\text { normalisation }\end{array}$ & $\begin{array}{c}\text { (Li et al., } \\
\text { 2017b) }\end{array}$ & $\begin{array}{l}\text { Synopsys } \\
\text { Design } \\
\text { Compiler }\end{array}$ & -0.02 & -83.8 & 88.9 & $\begin{array}{l}\text { Binary } \\
\text { computing }\end{array}$ \\
\hline $\mathrm{SC} \mathrm{MAC}$ & $\begin{array}{c}\text { (Sim \& Lee, } \\
\text { 2017) }\end{array}$ & $\begin{array}{c}\text { Synopsys } \\
\text { Design } \\
\text { Compiler } \\
\end{array}$ & -1 & -93.9 & -89.4 & $\begin{array}{l}\text { Binary } \\
\text { computing } \\
\text { ASIC* }\end{array}$ \\
\hline \multirow[t]{4}{*}{ SC DMAC } & \multirow{4}{*}{$\begin{array}{l}\text { (Hojabr et } \\
\text { al., 2019) }\end{array}$} & \multirow{4}{*}{$\begin{array}{l}\text { Synopsys } \\
\text { Design } \\
\text { Compiler }\end{array}$} & \multirow[t]{4}{*}{-} & \multirow[t]{4}{*}{-73.5} & 292 & AlexNet \\
\hline & & & & & 147 & InceptionV3 \\
\hline & & & & & 370 & VGG16 \\
\hline & & & & & 12 & MobileNet \\
\hline $\begin{array}{c}\text { SC Sigmoid } \\
\text { activation }\end{array}$ & $\begin{array}{c}\text { (Li et al., } \\
2017 \mathrm{a})\end{array}$ & FreePDK & -0.01 & -96.8 & 96.7 & $\begin{array}{l}\text { Binary } \\
\text { computing }\end{array}$ \\
\hline \multirow[t]{3}{*}{$\begin{array}{c}\text { SC } \\
\text { Quantization }\end{array}$} & \multirow[t]{3}{*}{$\begin{array}{l}\text { (Li et al., } \\
\text { 2018b) }\end{array}$} & \multirow[t]{3}{*}{ FreePDK } & -0.79 & -98.6 & 99.1 & $\begin{array}{l}\text { Binary } \\
\text { computing }\end{array}$ \\
\hline & & & - & -45.7 & 77.9 & $\begin{array}{l}\text { Unipolar } \\
\text { SC }\end{array}$ \\
\hline & & & - & -60.3 & 85.8 & Bipolar SC \\
\hline SC BNN & $\begin{array}{c}\text { (Hirtzlin et } \\
\text { al., 2019) }\end{array}$ & $\begin{array}{c}\text { Cadence } \\
\text { First } \\
\text { Encounter }\end{array}$ & -1.40 & -62.0 & 240 & $\begin{array}{l}\text { Binary } \\
\text { BNN }\end{array}$ \\
\hline
\end{tabular}

*Binary computing ASIC apply to the CNN model comparison. 